\title{
4-Aminopyrimidine-5-carbaldehydes as intermediates in a Friedländer type synthesis of 7-arylpyrido[2,3-d]pyrimidines
}

\author{
Jairo Quiroga, ${ }^{a^{*}}$ Jorge Trilleras, ${ }^{b}$ Rodrigo Abonía, ${ }^{a}$ Braulio Insuasty, ${ }^{a}$ Manuel Nogueras, ${ }^{c^{*}}$ \\ Justo Cobo, ${ }^{\mathrm{c}}$ and José M. de la Torre ${ }^{\mathrm{c}}$ \\ ${ }^{a}$ Grupo de Investigación de Compuestos Heterocíclicos, Departamento de Química, Universidad \\ del Valle, A. A. 25360, Cali, Colombia \\ ${ }^{b}$ Grupo de Investigación de Compuestos Heterocíclicos, Departamento de Química, Universidad \\ del Atlántico, Barranquilla, Colombia \\ ${ }^{c}$ Department of Inorganic and Organic Chemistry, Universidad de Jaén, 23071 Jaén, Spain \\ E-mail: mmontiel@ujaen.es,jaiquir@univalle.edu.co
}

\begin{abstract}
A study of formylation of 6-aminopyrimidines leads to the conclusion that the formylation at C5 occurs only when there is no contribution of heteroaromaticity in the pyrimidine ring and that the corresponding pyrimidoformamides are formed in heteroaromatic pyrimidines. Once 4aminopyrimidin-4(3H)-one-5-carboxaldehydes were prepared, a series of 7-arylpyrido[2,3$d$ ]pyrimidines derivatives were synthesized by a Friedländer type reaction with acetophenones under solvent-free conditions and in the presence of $\mathrm{BF}_{3}-\mathrm{Et}_{2} \mathrm{O}$. The yields of 7-arylpyrido[2,3$d$ ]pyrimidines range from moderate to good and the reaction times were quite short.
\end{abstract}

Keywords: Nitrogenated heterocycles, acylation, cyclocondensation, solvent free, regioselectivity

\section{Introduction}

Nitrogen heterocycles have received a great deal of attention in the literature as a result of their role as pharmacophores of great historical significance. Among these heterocyclic systems, especially those containing pyridine have been the subject of expanding research efforts in heteroaromatic and biological chemistry. ${ }^{1}$ The pyrido[2,3- $d$ ]pyrimidine heterocycles (a.k.a. 5deazapteridines), which are those annelated to a pyrimidine ring, are of paramount importance because of their wide range of biological $^{1 \mathrm{a}, 2}$ and pharmaceutical applications (i.e., bronchodilators, vasodilators) and their anti-allergic, cardiotonic, antihypertensive, and hepatoprotective activities. ${ }^{3}$ Some of them have shown properties as antitumour, antibacterial, analgesic or CNS depressants. ${ }^{4}$ 
The synthesis of pyrido[2,3- $d$ ]pyrimidines has been typically performed by annelation of the pyrimidine ring on the pyridine or vice versa, ${ }^{5}$ and these methods include diverse procedures based on the Michael addition-cyclodehydration strategy, ${ }^{1,5 f}$ the three-component one-pot synthesis, ${ }^{2,6}$ the route via the aza-Wittig reaction ${ }^{7}$ from iodouracils, having a formamidine or acetamidine moiety with various acetylene derivatives. ${ }^{8}$ Aside from the traditional methodologies, more recent methods include a microwave-assisted one-pot synthesis ${ }^{3,4 c}$ and a procedure that involves ultrasound irradiation without a catalyst. ${ }^{9}$ Although, the synthesis of this bicyclic system is well documented, most of the synthetic methods rely on cyclocondensation that usually require drastic conditions, long reaction times and complex synthetic pathways. Thus, the search for new, fast routes of synthesis of this kind of molecules remains of considerable importance.

In the course of our synthetic studies of simple nitrogen-containing heterocycles, we have already reported the synthesis of functionalized pyrido[2,3- $d$ ]pyrimidines via a selective cyclocondensation reaction between 6-aminopyrimidines and Mannich bases $(\beta$ dimethylaminopropiophenone hydrochlorides), 3-formylchromone, 2-dimethylaminomethylentetralone hydrochloride, chalcones and methylene-active compounds, ${ }^{10}$ and also by using microwaveassisted multicomponent synthesis. ${ }^{11}$

On the other hand, the Friedländer reaction is a well-known method for the preparation of heteroannulated compounds, ${ }^{12}$ and we consider that it is one of the most useful methods for the preparation of pyridopyrimidines and related bicyclic systems. This consists of the reaction between an aromatic or heteroaromatic ortho-amino-carbaldehyde and an aldehyde or ketone bearing an $\alpha$-methylene moiety. In most cases, this cyclocondensation reaction type was carried out under conventional methods and with longer reaction times. In this way, the possibility of performing reactions under solvent-free conditions is particularly attractive because of its simplicity, lower environmental impact, and minimum reaction time, which can provide a rapid access to large libraries of diverse molecules.

Here we provide a simple and solvent-free method for the preparation of 7-arylpyrido[2,3$d$ ]pyrimidines from 6-aminopyrimidines. We have previously prepared the corresponding 6aminopyrimidin-5-carboxaldehydes in order to act as intermediates in a Friedländer reaction type. $^{13}$

\section{Results and Discussion}

We have tried the preparation of 4-aminopyrimidin-5-carboxaldehydes from 6-aminopyrimidin$4(3 H)$-ones 2 a-d. Two classical formylation reagents, the acetic anhydride/formic acid mixture ${ }^{14}$ or the Vilsmeier reagent, ${ }^{15}$ have been used (see Scheme 1). The reaction of the 6aminopyrimidines with an acetic anhydride/formic acid mixture led in all cases to the corresponding 6-formamidepyrimidine derivatives $\mathbf{4}$ in good to excellent yields. 
On the other hand, the use of Vilsmeier's reagent rendered different results depending on the starting material, reaction conditions and work-up (Scheme 1). Thus, the formylation at C-5 in the pyrimidine nucleus was effective only when the 3-methyl derivatives 2a,b were used. In the case of the $3 \mathrm{H}$ analogues $\mathbf{2 c , d}$, the different attempts of formylation at C5 with Vilsmeier's reagent resulted in all cases in attack at the 6-amino group. Depending on the work-up conditions, one may i) recover the starting material 2c,d, ii) isolate the corresponding amidoderivatives $\mathbf{4 c}, \mathbf{d}$ or iii) produce the ( $N, N$-dimethyl)amidine derivative 3c (on work-up with ethanol at room temperature for $48 \mathrm{~h}$ ).

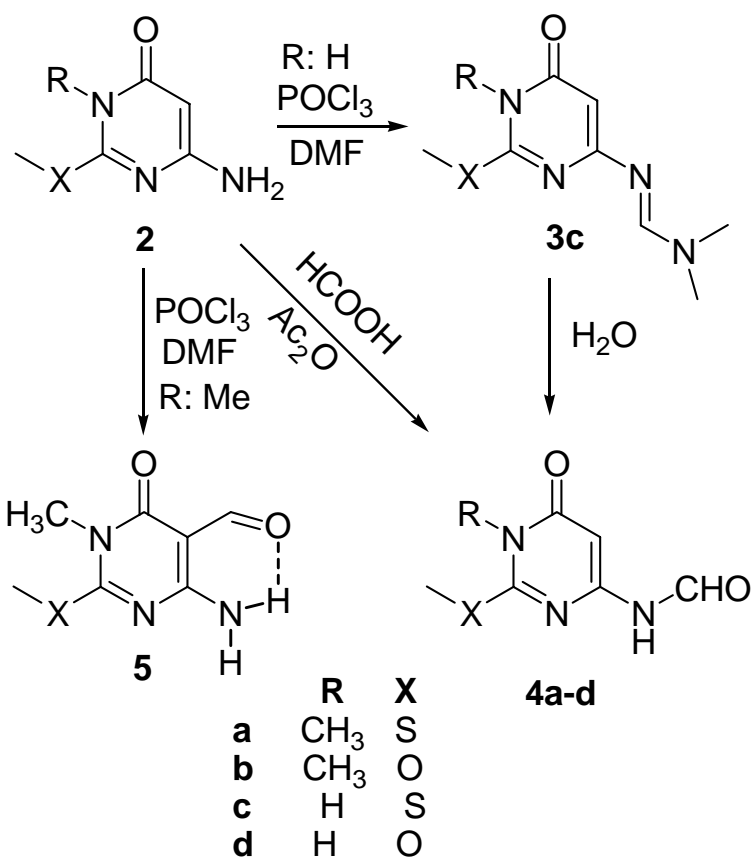

Scheme 1. Formylation reactions of 6-aminopyrimidin-4(3H)-ones 2.

This marked difference in the behaviour of the $\mathrm{N}(3)-\mathrm{CH}_{3} v s . \mathrm{N}(3)-\mathrm{H}$ pyrimidines, 2a,b vs. 2c,d, respectively, is due to the nucleophilic characters of $\mathrm{C} 5$ and the 6-amino group. For 2a,b that amino group is part of an amide vinylogous moiety and C5 is the most nucleophilic site, while for $\mathbf{2 c , d}$, the possibility of lactam-lactim tautomerism gives this compound some heteroaromatic character and results in a higher nucleophilic character of the amino group. In fact, the use of Vilsmeier conditions to the heteroaromatic 6-chloro-2-(methylthio)pyrimidin-4-amine, 2e, or 4,6-dimethoxypyrimidin-2-amine, $\mathbf{2 f}$, affords reaction at the amino group and yields $\mathbf{3 e}$ and $\mathbf{4 f}$, respectively (Figure 1). 

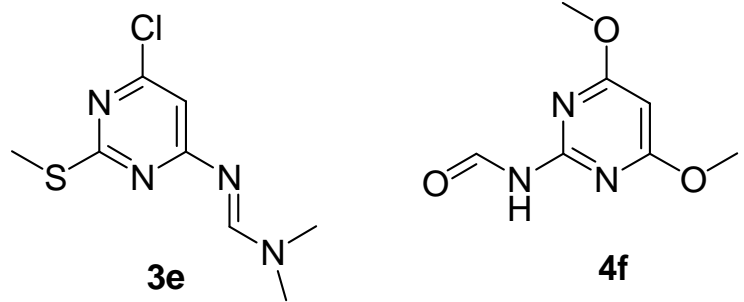

Figure 1. Compounds isolated by Vilsmeier formylation of 6-amino-4-chloro-2methylthiopyrimidine 2e and 2-amino-4,6-dimethoxypyrimidine $\mathbf{2 f}$.

Optimized conditions for the preparation of the 5-formylpyrimidine 5a (Scheme 2) involved warming to $50{ }^{\circ} \mathrm{C}$ for $18 \mathrm{~h}$, quenching with ice and neutralizing with $\mathrm{NaOH}$, and a second heating for $1 \mathrm{~h}$, and this protocol resulted in a $88 \%$ yield. The application of this protocol to the preparation of the 6-amino-1,3-dimethyl-2,4-dioxo-1,2,3,4-tetrahydropyrimidine-5-carbaldehyde $\mathbf{1}$ resulted in a $98 \%$ yield; an improvement over the previously reported $90 \%$ yield. ${ }^{16}$ For the $2-$ methoxypyrimidine $\mathbf{2 b}$, the reaction proceeded under such conditions with hydrolysis of the methoxy group and formylation at both $\mathrm{C} 5$ and $6-\mathrm{NH}_{2}$ giving $\mathbf{6 g}$ (86\% yield), which can be hydrolyzed in aqueous $\mathrm{NaOH}$ to afford the known 6-amino-3-methyl-2,4-dioxo-1,2,3,4tetrahydro-5-pyrimidine-carbaldehyde ${ }^{17} \mathbf{3 g}$ in $84 \%$ yield (Scheme 2 ). Thus, in order to prepare the 5 -formyl derivative $\mathbf{5 b}$ from $\mathbf{2 b}$, the reaction with the Vilsmeier reagent was carried out at 0 ${ }^{\circ} \mathrm{C}$ to afford $\mathbf{5 b}$ in $70 \%$ yield.

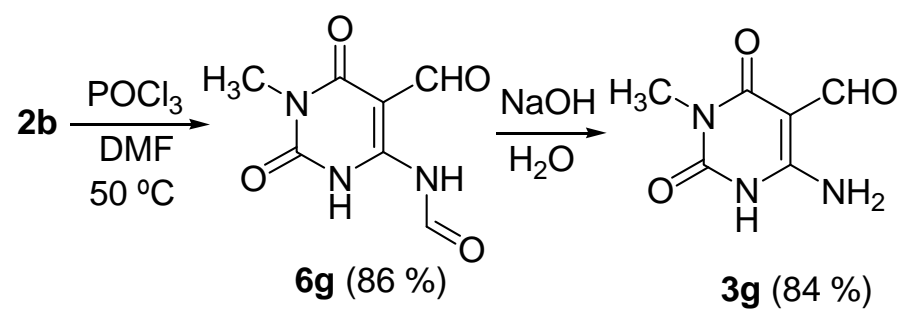

Scheme 2. Formylation of 6-amino-2-methoxy-3-methylpyrimidin-4(3H)-one $2 \mathbf{b}$ under the optimized conditions used in the preparation of compound $5 \mathbf{a}$ from $\mathbf{2 a}$.

The ${ }^{1} \mathrm{H}$ NMR spectra of $\mathbf{1}, \mathbf{5 a}, \mathbf{b}$ and $\mathbf{3 g}$ show two broad singlets for the protons of the 4- $\mathrm{NH}_{2}$ group due to the intramolecular hydrogen bond between the $\mathrm{H}$ and the oxygen atom of the 5CHO group (Experimental and Scheme 1). 4-Aminopyrimidine-5-carbaldehydes 1, 5a,b were reacted with acetophenones 7 under different conditions. The use of an equimolar ratio and catalytic amount of $\mathrm{BF}_{3}-\mathrm{Et}_{2} \mathrm{O}$ (3 drops) through a solvent-free fusion method was appropriate to render the desired 7-arylpyrido[2,3- $d$ ] pyrimidines $\mathbf{8}$ - 10 in moderate to good yields within a few seconds. The pyridopyrimidines were filtrated and purified by recrystallization or by column chromatography. 
To the best of our knowledge, $\mathrm{BF}_{3}-\mathrm{Et}_{2} \mathrm{O}$ has been used in the syntheses of several chalcones through a Claisen-Schmidt reaction. ${ }^{18}$ Here the 7-arylpyrido[2,3- $d$ ]pyrimidines were obtained through the initial formation of the corresponding chalcones by an acid-catalyzed ClaisenSchmidt reaction and subsequent intramolecular cyclocondensation between amino and carbonyl groups to give 7-arylpyrido[2,3- $d$ ]pyrimidines $\mathbf{8}$ - 10 (Scheme 3). It is important to note that $\mathrm{BF}_{3}$ $\mathrm{Et}_{2} \mathrm{O}$ can be used in the presence of ester and amide functional groups. The use of basic catalytic conditions resulted in hydrolysis. On the contrary, the thermal cyclization of compounds $\mathbf{5 b}$ occurs through the hydrolysis of the 2-methoxy group to form $\mathbf{1 0}$.

Something similar happened in other previous acid reactions in presence of a nucleophile as water formed in the condensation step. ${ }^{19}$ Compounds $\mathbf{1 0}$, which are difficult to produce from the corresponding 6-amino-3-methyluracil, can also be obtained from the 6-amino-3-methyl-2,4dioxo-1,2,3,4-tetrahydro-5-pyrimidinecarbaldehyde 3g.

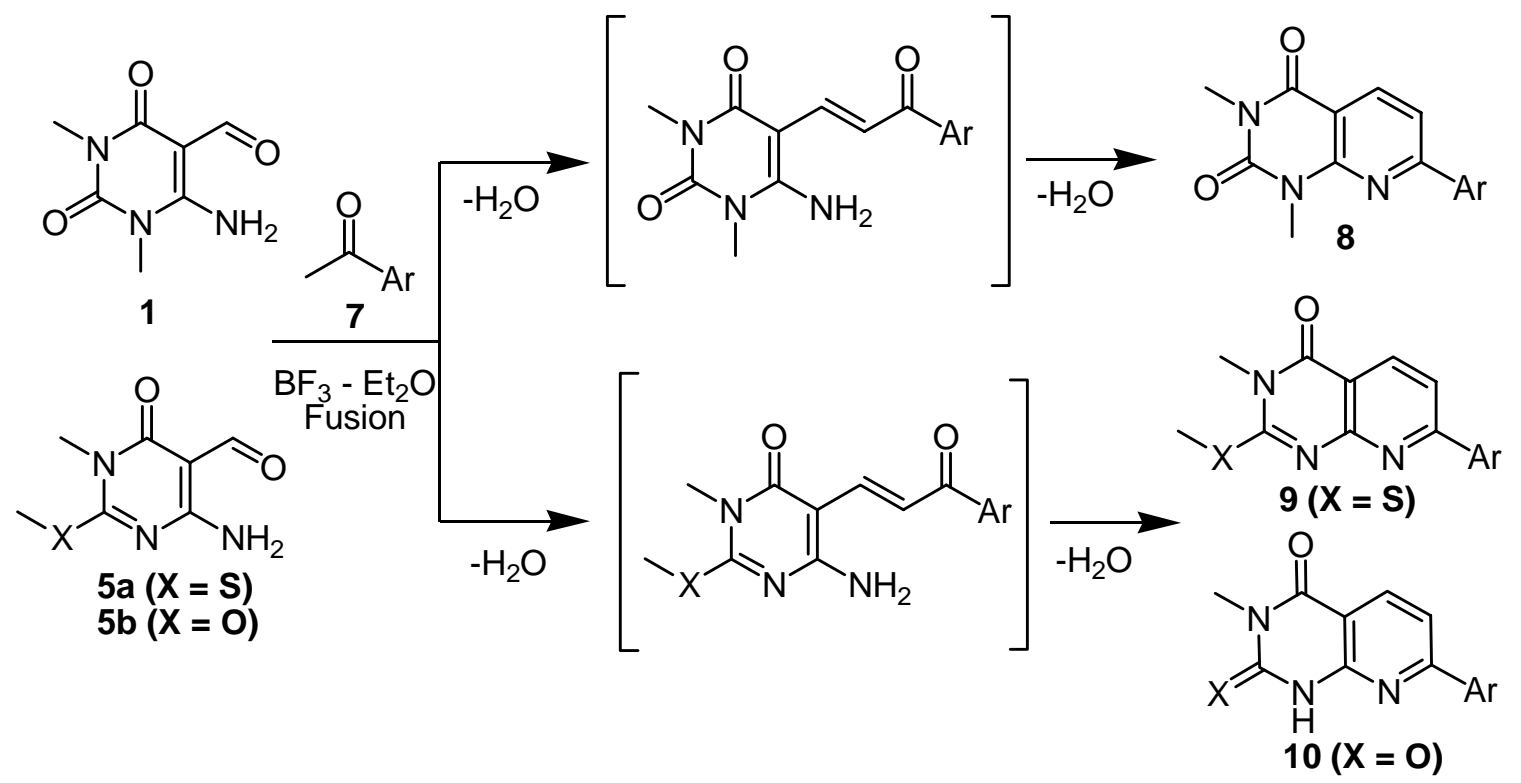

Scheme 3. Solvent-free reaction of 6-aminopyrimidine-5-carbaldehydes and acetophenones to 7arylpyrido[2,3- $d]$ pyrimidines. 
Table 1. Solvent-free preparation of 7-arylpyrido[2,3- $d$ pyrimidines: Melting points $\left({ }^{\circ} \mathrm{C}\right)$ and yields

\begin{tabular}{|c|c|c|c|c|c|c|c|}
\hline \multirow[t]{2}{*}{ Entry } & \multirow[t]{2}{*}{$\mathrm{Ar}$} & \multicolumn{2}{|c|}{ Compound $\mathbf{8}$ from $\mathbf{1}$} & \multicolumn{2}{|c|}{$\begin{array}{c}\text { Compound } 9 \\
\text { from } 5 \mathbf{a}\end{array}$} & \multicolumn{2}{|c|}{$\begin{array}{l}\text { Compound } 10 \\
\text { from } 5 \mathbf{b}\end{array}$} \\
\hline & & M.p. ${ }^{a}$ & $\%$ & M.p. & $\%$ & M.p. & $\%$ \\
\hline $\mathbf{a}$ & $\mathrm{C}_{6} \mathrm{H}_{5}$ & $157-159(156-157)$ & 65 & $129-131$ & 75 & 291-293 & 70 \\
\hline b & $4-\mathrm{H}_{3} \mathrm{CC}_{6} \mathrm{H}_{4}$ & $175-177(173-174)$ & 50 & $189-191$ & 70 & $>300$ & 60 \\
\hline c & $4-\mathrm{H}_{3} \mathrm{COC}_{6} \mathrm{H}_{4}$ & 163-165 (164) & 65 & 196-198 & 70 & $>300$ & 70 \\
\hline d & $\begin{array}{c}3,4,5- \\
\left(\mathrm{H}_{3} \mathrm{CO}\right)_{3} \mathrm{C}_{6} \mathrm{H}_{2}\end{array}$ & 211-213 & 40 & 211-213 & 70 & 297-299 & 60 \\
\hline e & $4-\mathrm{C}_{6} \mathrm{H}_{5} \mathrm{C}_{6} \mathrm{H}_{4}$ & 233-235 & 60 & $222-224$ & 50 & $>300$ & 40 \\
\hline $\mathbf{f}$ & $4-\mathrm{HOC}_{6} \mathrm{H}_{4}$ & >300 dec. & 40 & $249-251$ & 80 & $>300$ & 60 \\
\hline g & $4-\mathrm{O}_{2} \mathrm{NC}_{6} \mathrm{H}_{4}$ & 283-285 & 50 & $272-274$ & 70 & -- & -- \\
\hline h & $4-\mathrm{ClC}_{6} \mathrm{H}_{4}$ & $138-140(139-140)$ & 40 & $219-221$ & 70 & 313-315 & 60 \\
\hline $\mathbf{i}$ & $4-\mathrm{BrC}_{6} \mathrm{H}_{4}$ & 217-219 & 40 & 206-208 & 80 & $310-312$ & 50 \\
\hline $\mathbf{j}$ & 4-FC ${ }_{6} \mathrm{H}_{4}$ & 207-209 & 60 & 202-204 & 70 & 295-297 & 60 \\
\hline k & Coumaryl & 284-286 & 60 & 217-219 & 70 & -- & -- \\
\hline
\end{tabular}

${ }^{a}$ M.p. reported by Troschuz et al..$^{5 f}$ are given in parentheses.

The structures of compounds $\mathbf{8 , 9}$ and $\mathbf{1 0}$ were confirmed by spectroscopic analysis. All products show NMR spectra that are in agreement with the proposed structures. The assignment of carbon atoms was based on DEPT-135 measurements along with 2-dimensional HMBC and HSQC $\left({ }^{1} \mathrm{H}^{13} \mathrm{C}\right)$ experiments. All sets of compounds 8 and $\mathbf{9}$ present similar spectroscopic profiles. For example, in the ${ }^{1} \mathrm{H}$ NMR spectrum of compound 8a the signals of H-6 and H-5 in the pyridine ring appear at 7.66 and $8.49 \mathrm{ppm}$ respectively as two doublets with $J_{\text {ortho }}=8.03 \mathrm{~Hz}$, and in addition to the heteronuclear correlation observed in the HMBC experiment between signal assigned to $\mathrm{C}(4)$ with those to $\mathrm{N}(3)-\mathrm{CH}_{3}$ and $\mathrm{H}-5$.

Regarding compounds $\mathbf{1 0}$ the spectroscopic data confirm the hydrolysis of the methoxy group at $\mathrm{C}(2)$, the corresponding signal for the $\mathrm{H}-1$ proton of the pyrimidine ring. Finally, the structure of the 7-arylpyrido[2,3- $d$ ]pyrimidines was unambiguously confirmed by X-ray diffraction analysis of compounds $\mathbf{8 b}, \mathbf{8 e}, \mathbf{1 0 c}$ and $\mathbf{1 0 j} .{ }^{20}$

\section{Conclusions}

We have shown that formylation of 6-aminopyrimidines can occur at the amino group in heteroaromatic pyrimidine systems, and it can occur at C5 if the heteroaromaticity of the pyrimidine ring is low. Using these 5-formylpyrimidine derivatives we have developed a new, rapid and simple methodology for the synthesis of several functionalized pyrido[2,3- 
d]pyrimidines of potential biological importance in moderate to good yields. This method can be very useful for high-throughput synthesis. A particularly valuable feature of this method consists in the fact that, for the first time, the reaction was catalysed by $\mathrm{BF}_{3}-\mathrm{Et}_{2} \mathrm{O}$. The advantages of our method compared to existing methods are short reaction times, solvent-free conditions, low cost and high tolerance of sensitive functional groups such as esters or amides.

\section{Experimental Section}

General. Melting points were determined in a Buchi Melting Point Apparatus and are reported uncorrected. The ${ }^{1} \mathrm{H}$ - and ${ }^{13} \mathrm{C}$ NMR spectra were measured on Bruker Avance 400 spectrometers operating at 400 and $100 \mathrm{MHz}$, respectively, and using DMSO- $d_{6}$ as solvent and tetramethylsilane as internal standard. The mass-spectra were scanned on a Hewlett Packard HP Engine-5989 spectrometer (equipped with a direct inlet probe) which was operating at $70 \mathrm{eV}$. High Resolution Mass Spectra (HRMS) were recorded in a Waters Micromass AutoSpec NT spectrometer (STIUJA). The elemental analyses have been obtained using a LECO CHNS-900 and Thermo Finnigan FlashEA1112 CHNS-O (STIUJA) elemental analyzers.

\section{General procedure A for the synthesis of $N$-pyrimidin-4-yl formamides with acetic anhydride/formic acid mixture}

$0.5 \mathrm{ml}$ acetic anhydride, $0.15 \mathrm{ml}$ formic acid and $1 \mathrm{mmol}$ of the corresponding 6aminopyrimidine were warmed for the specific time and temperature for each starting aminopyrimidine. The reaction was monitored by TLC (eluent $\mathrm{CH}_{2} \mathrm{Cl}_{2}-\mathrm{MeOH}, 9: 1$ ). After reaction of all the aminopyrimidine substrate, the mixture was dried under reduce pressure and the crude product was recrystallized from EtOH. The solid was filtered and washed with cool EtOH.

\section{General procedure B for Vilsmeier formylation of 6-Aminopyrimidines}

$0.15 \mathrm{ml} \mathrm{POCl}_{3}$ in $0.5 \mathrm{ml}$ DMF was stirred in an ice bath for 15 minutes. Then, another $1.5 \mathrm{ml}$ DMF and $1 \mathrm{mmol}$ of the corresponding 6-aminopyrimidine were added to the cool solution. The reaction takes place at the corresponding temperature and time for each case. The reaction was monitored by TLC (eluent $\mathrm{CH}_{2} \mathrm{Cl}_{2}-\mathrm{MeOH}$, 9:1). After complete comsumption of the aminopyrimidine substrate, the mixture was poured into crushed ice and the solution was allowed to warm to room temperature and then brought to a boil and neutralized with $\mathrm{NaOH}$. The heating continues for an hour and let it cooled to room temperature. The resulting precipitated was filtered, washed with water and recrystallized from EtOH.

6-Amino-1,3-dimethyl-2,4-dioxo-1,2,3,4-tetrahydropyrimidine-5-carbaldehyde 1. $0.155 \mathrm{~g}$ of 6-amino-1,3-dimethylpyrimidine-2,4(1H,3H)-dione reacts according to the general procedure $\mathrm{B}$ for 30 minutes at $50^{\circ} \mathrm{C}$. Yield of $98 \%$, mp $195-198{ }^{\circ} \mathrm{C}$, bibliographic mp $196-198{ }^{\circ} \mathrm{C} .{ }^{16}$ 
$N, N$-Dimethyl- $N$ '-[2-(methylthio)-6-oxo-1,6-dihydropyrimidin-4-yl]imidoformamide 3c. $0.157 \mathrm{~g}$ of 6-amino-2-(methylthio)pyrimidin-4(3H)-one reacts according to the general procedure $\mathrm{B}$ at $0^{\circ} \mathrm{C}$ for 7 hour. White solid. Yield 60\%, mp 161-162 ${ }^{\circ} \mathrm{C}$. ${ }^{1} \mathrm{H}$ NMR (400 MHz, DMSO- $\left.d_{6}\right): \delta=$ 2.56 (s, 3H, $\left.\mathrm{SCH}_{3}\right) ; 3.41$ (s, 3H, $\mathrm{NCH}_{3}$ ); 3.43 (s, 3H, $\left.\mathrm{NCH}_{3}\right) ; 6.38$ (s, 1H, C $\left.5-\mathrm{H}\right) ; 8.92$ (s, 1H, $\mathrm{CH}) ; 12.48$ (s, $1 \mathrm{H}, \mathrm{NH}) .{ }^{13} \mathrm{C}$ NMR $\left(100 \mathrm{MHz}, \mathrm{DMSO}-d_{6}\right): \delta=13.15\left(\mathrm{SCH}_{3}\right) ; 39.07\left(\mathrm{NCH}_{3}\right)$; $44.54\left(\mathrm{NCH}_{3}\right) ; 92.81\left(\mathrm{C}_{5} \mathrm{H}\right) ; 152.05(\mathrm{CH}) ; 154.73\left(\mathrm{C}_{6}\right) ; 164.26\left(\mathrm{C}_{4}\right) ; 164.94\left(\mathrm{C}_{2}\right)$. IR $(\mathrm{KBr}) \mathrm{cm}^{-1}$ $3004(\mathrm{NH} s t), 1646(\mathrm{C}=\mathrm{O} s t) 1594(\mathrm{NH} \delta) . \mathrm{MS}(70 \mathrm{eV}) \mathrm{m} / z(\%) 212\left(\mathrm{M}^{+}, 42\right), 197$ (28), 156 (12), 68 (25), 42 (100). HR-MS calc. for $\mathrm{C}_{8} \mathrm{H}_{12} \mathrm{~N}_{4} \mathrm{OS} 212.0725$ found 212.0732.

$N^{\prime}$-[6-Chloro-2-(methylthio)pyrimidin-4-yl]- $N, N$-dimethylimidoformamide 3e. 0.175 g of 6chloro-2-(methylthio)pyrimidin-4-amine, 2e, reacts according to the general procedure B with the Vilsmeier's reagent for 1 hour and 30 minutes at $50^{\circ} \mathrm{C}$. White solid, yield $61 \%$, mp 98-99 ${ }^{\circ} \mathrm{C}$. ${ }^{1} \mathrm{H}$ NMR (400 MHz, DMSO- $d_{6}$ ): $\delta=2.46$ (s, 3H, $\mathrm{SCH}_{3}$ ); 3.02 (s, 3H, $\mathrm{NCH}_{3}$ ); 3.15 (s, 3H, $\left.\mathrm{NCH}_{3}\right) ; 6.52$ (s, 1H, C $\left.5-\mathrm{H}\right) ; 8.66(\mathrm{~s}, 1 \mathrm{H}, \mathrm{CH}) .{ }^{13} \mathrm{C}$ NMR (100 MHz, DMSO-d $\left.d_{6}\right): \delta=13.56$ $\left(\mathrm{SCH}_{3}\right) ; 34.76\left(\mathrm{NCH}_{3}\right) ; 40.84\left(\mathrm{NCH}_{3}\right) ; 107.92\left(\mathrm{C}_{5}\right) ; 157.70(\mathrm{CH}) ; 158.91\left(\mathrm{C}_{6}\right) ; 168.36\left(\mathrm{C}_{4}\right)$; $170.82\left(\mathrm{C}_{2}\right)$. MS (70 eV) m/z (\%) $230\left(\mathrm{M}^{+}, 100\right), 215$ (18), 184 (18), 149 (31). HR-MS calc. for $\mathrm{C}_{8} \mathrm{H}_{11} \mathrm{ClN}_{4} \mathrm{~S} 230.0390$ found 230.0393. Anal. Calcd for $\mathrm{C}_{8} \mathrm{H}_{11} \mathrm{ClN}_{4} \mathrm{~S}$ : C, 41.65; H, 4.99; N, 24.28; S, 13.90. Found: C, 42.14; H, 4.99; N, 24.30; S, 14.11.

$\mathrm{N}$-[1-Methyl-2-(methylthio)-6-oxo-1,6-dihydropyrim-idin-4-yl]formamide 4a. $0.174 \mathrm{~g}$ of 6amino-3-methyl-2-(methylthio)pyrimidin-4(3H)-one reacts according to the general procedure A at $50{ }^{\circ} \mathrm{C}$ for 10 minutes. White solid. Yield 75\%, mp 255-258 ${ }^{\circ} \mathrm{C}$. ${ }^{1} \mathrm{H}$ NMR (400 MHz, DMSO-

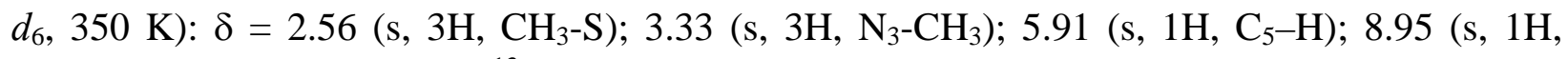
$\mathrm{CHO}) ; 10.28$ (s, $\left.1 \mathrm{H}, \mathrm{C}_{4}-\mathrm{NH}\right) .{ }^{13} \mathrm{C}$ NMR $\left(100 \mathrm{MHz}, \mathrm{DMSO}-d_{6}, 350 \mathrm{~K}\right): \delta=14.13\left(\mathrm{~S}-\mathrm{CH}_{3}\right) ; 29.23$ $\left(\mathrm{N}-C H_{3}\right) ; 89.57\left(\mathrm{C}_{5}\right)$; $163.22\left(\mathrm{C}_{2}\right) ; 153.42\left(\mathrm{C}_{6}\right) ; 161.19\left(\mathrm{C}_{4}\right) ; 161.30(\mathrm{CHO}) . \mathrm{IR}(\mathrm{KBr}) \mathrm{cm}^{-1} 3004$ (NH st), 1646 (C=O st) $1594\left(\mathrm{NH} \delta\right.$ ). MS (70 eV) m/z (\%) $199\left(\mathrm{M}^{+}, 39\right) ; 171$ (64); 68 (64); 57 (100). HR-MS calc. for $\mathrm{C}_{7} \mathrm{H}_{9} \mathrm{~N}_{3} \mathrm{O}_{2} \mathrm{~S} 199.0415$ found 199.0420. Anal. Calcd for $\mathrm{C}_{7} \mathrm{H}_{9} \mathrm{~N}_{3} \mathrm{O}_{2} \mathrm{~S}$ : C, 42.25; H, 4.52; N, 21.10; S, 16.08. Found: C, 41.94; H, 4.52; N, 20.77; S, 15.76.

$\mathrm{N}$-(2-Methoxy-1-methyl-6-oxo-1,6-dihydropyrimidin-4-yl)formamide $4 \mathbf{b}$. 0.155 g of 6amino-2-methoxy-3-methylpyrimidin-4(3H)-one reacts according to the general procedure $\mathrm{A}$ at $50{ }^{\circ} \mathrm{C}$ for 15 minutes. White solid. Yield 90\%, mp 214-215 ${ }^{\circ} \mathrm{C}$. ${ }^{1} \mathrm{H}$ NMR (400 MHz, DMSO- $d_{6}$, $350 \mathrm{~K}$ ): $\delta=3.24$ (s, 3H, N3- $\mathrm{CH}_{3}$ ); 4.00 (s, 3H, $\mathrm{CH}_{3}-\mathrm{O}$ ); 5.82 (s, 1H, $\mathrm{C}_{5}-\mathrm{H}$ ); 8.94 (s, 1H, CHO); 10.24 (s, $\left.1 \mathrm{H}, \mathrm{C}_{4}-\mathrm{NH}\right) .{ }^{13} \mathrm{C}$ NMR $\left(100 \mathrm{MHz}, \mathrm{DMSO}-d_{6}, 350 \mathrm{~K}\right): \delta=: 26.62\left(\mathrm{~N}_{-} \mathrm{CH}_{3}\right) ; 55.46(\mathrm{O}-$ $\left.\mathrm{CH}_{3}\right) ; 90.32\left(\mathrm{C}_{5}\right) ; 156.91\left(\mathrm{C}_{2}\right) ; 154.98\left(\mathrm{C}_{6}\right) ; 158.54\left(\mathrm{C}_{4}\right) ; 162.45(\mathrm{CHO}) . \mathrm{IR}(\mathrm{KBr}) \mathrm{cm}^{-1} 3377$ (NH st), $1616(\mathrm{C}=\mathrm{O} s t) 1512\left(\mathrm{NH} \delta\right.$ ). MS (70 eV) m/z (\%) $183\left(\mathrm{M}^{+}, 7\right) ; 155$ (27); 125 (6); 40 (100). HR-MS calc. for $\mathrm{C}_{7} \mathrm{H}_{9} \mathrm{~N}_{3} \mathrm{O}_{3} 183.0644$ found 183.0645. Anal. Calcd for $\mathrm{C}_{7} \mathrm{H}_{9} \mathrm{~N}_{3} \mathrm{O}_{3}$ : C, 45.94; H, 4.92; N, 22.95. Found: C, 45.65; H, 5.15; N, 23.03.

$\mathrm{N}$-[2-(Methylthio)-6-oxo-1,6-dihydropyrimidin-4-yl]formamide 4c. $0.157 \mathrm{~g}$ of 6-amino-2(methylthio)pyrimidin-4(3H)-one reacts according to the general procedure A at $50^{\circ} \mathrm{C}$ for 30 minutes. White solid. Yield 69\%, mp 290-291 ${ }^{\circ} \mathrm{C}$. ${ }^{1} \mathrm{H}$ NMR (400 MHz, DMSO- $\left.d_{6}, 350 \mathrm{~K}\right): \delta=$ 2.50 (s, 3H, $\mathrm{CH}_{3}-\mathrm{S}$ ); 5.94 (s, 1H, C $5-\mathrm{H}$ ); 8.95 (s, 1H, CHO); 10.59 (s, 1H, C4-NH); 12.44 (s, 1H, $\left.\mathrm{N}_{1}-\mathrm{H}\right) .{ }^{13} \mathrm{C}$ NMR $\left(100 \mathrm{MHz}, \mathrm{DMSO}-d_{6}, 350 \mathrm{~K}\right): \delta=12.49\left(\mathrm{~S}_{-} \mathrm{CH}_{3}\right) ; 89.87\left(\mathrm{C}_{5}\right) ; 169.60\left(\mathrm{C}_{2}\right)$; 
$156.03\left(\mathrm{C}_{6}\right) ; 164.40\left(\mathrm{C}_{4}\right) ; 161.33(\mathrm{CHO})$. IR (KBr) $\mathrm{cm}^{-1} 3217(\mathrm{NH} s t), 1645,1684(\mathrm{C}=\mathrm{O} s t) 1515$ $(\mathrm{NH} \delta$ ). MS (70 eV) m/z (\%) 185 (M+1 60); 157 (86); 110 (25); 74 (29); 68 (100). HR-MS calc. for $\mathrm{C}_{6} \mathrm{H}_{7} \mathrm{~N}_{3} \mathrm{O}_{2} \mathrm{~S} 185.0259$ found 185.0257. Anal. Calcd for $\mathrm{C}_{6} \mathrm{H}_{7} \mathrm{~N}_{3} \mathrm{O}_{2} \mathrm{~S}$ : C, 38.95; H, 3.78; N, 22.70; S, 17.30. Found: C, 39.32; H, 3.96; N, 22.35; S, 16.61.

$\mathbf{N - ( 2 - M e t h o x y - 6 - o x o - 1 , 6 - d i h y d r o p y r i m i d i n - 4 - y l ) f o r m a m i d e ~ 4 d . ~} 0.141$ g of 6-amino-2methoxypyrimidin-4(3H)-one reacts according to the general procedure A at reflux for 2 hours. White solid. Yield 74\%, mp 226-228 ${ }^{\circ} \mathrm{C} .{ }^{1} \mathrm{H}$ NMR (400 MHz, DMSO- $d_{6}, 350 \mathrm{~K}$ ): $\delta=3.89$ (s, $3 \mathrm{H}, \mathrm{CH}_{3}-\mathrm{O}$ ); 5.77 (s, 1H, C5-H); 8.92 (s, 1H, CHO); 10.52 (s, 1H, C $\left.4-\mathrm{NH}\right) ; 12.04$ (s, 1H, N $-\mathrm{H}$ ). ${ }^{13} \mathrm{C}$ NMR (100 MHz, DMSO- $\left.d_{6}, 350 \mathrm{~K}\right): \delta=54.02\left(\mathrm{O}-\mathrm{CH}_{3}\right) ; 88.27\left(\mathrm{C}_{5}\right) ; 164.38\left(\mathrm{C}_{2}\right) ; 156.40$ $\left(\mathrm{C}_{6}\right) ; 158.56\left(\mathrm{C}_{4}\right) ; 161.31(\mathrm{CHO})$. IR (KBr) cm${ }^{-1} 3007$ (NH st), $1616(\mathrm{C}=\mathrm{O} s t) 1447(\mathrm{NH} \delta)$. MS (70 eV) m/z (\%) $169\left(\mathrm{M}^{+}, 25\right) ; 141$ (100); 111 (21); 68 (61). HR-MS calc. for $\mathrm{C}_{6} \mathrm{H}_{7} \mathrm{~N}_{3} \mathrm{O}_{3}$ 169.0487 found 169.0482. Anal. Calcd for $\mathrm{C}_{6} \mathrm{H}_{7} \mathrm{~N}_{3} \mathrm{O}_{3}$ : C, 67.40; H, 4.90; N, 15.72. Found: C, 67.70; H, 4.86; N, 15.26.

$\boldsymbol{N}$-(4,6-Dimethoxypyrimidin-2-yl)formamide 4 f. 0.155 g of 4,6-dimethoxypyrimidin-2-amine, 2f, reacts according to the general procedure $\mathrm{B}$ with the Vilsmeier's reagent at different temperatures and time reactions. For $0{ }^{\circ} \mathrm{C}$ and 17 hours of reaction, yield: $76 \%$. For $50{ }^{\circ} \mathrm{C}$ and 1 hour, yield: 72\%. White solid,.mp 124-126 ${ }^{\circ} \mathrm{C}$. ${ }^{1} \mathrm{H}$ NMR (400 MHz, DMSO- $\left.d_{6}\right): \delta=3.95$ (s, 6H, $\left.\mathrm{OCH}_{3}\right) ; 5.90$ (s, $\left.1 \mathrm{H}, \mathrm{C}_{5}-\mathrm{H}\right) ; 9.35$ (d, $\left.8.5 \mathrm{~Hz}, 1 \mathrm{H}, \mathrm{CHO}\right) ; 10.78$ (d, $\left.8.5 \mathrm{~Hz}, 1 \mathrm{H}, \mathrm{NH}\right) .{ }^{13} \mathrm{C}$ NMR (100 MHz, DMSO- $\left.d_{6}\right): \delta=54.10\left(\mathrm{OCH}_{3}\right) ; 84.11\left(\mathrm{C}_{5}\right) ; 156.83\left(\mathrm{C}_{2}\right) ; 163.50(\mathrm{CHO}) ; 171.58\left(\mathrm{C}_{4}\right.$ and $\left.\mathrm{C}_{6}\right)$. IR (KBr) cm ${ }^{-1}: 3134(\mathrm{NH} s t), 1697(\mathrm{C}=\mathrm{O} s t) 1401(\mathrm{NH} \delta)$. MS (70 eV) $\mathrm{m} / z(\%) 183$ ( $\left.\mathrm{M}^{+}, 22\right), 154$ (100), 125 (25), 68 (29). HR-MS calc. for $\mathrm{C}_{7} \mathrm{H}_{9} \mathrm{~N}_{3} \mathrm{O}_{3} 183.0642$ found 183.0644.

4-Amino-1-methyl-2-(methylthio)-6-oxo-1,6-dihydropyrimidine-5-carbaldehyde 5a. $0.174 \mathrm{~g}$ of 6-Amino-3-methyl-2-(methylthio)pyrimidin-4(3H)-one reacts according to the general procedure B for 18 hours at $50{ }^{\circ} \mathrm{C}$. White solid. Yield 88\%, mp 226-228 ${ }^{\circ} \mathrm{C} .{ }^{1} \mathrm{H}$ NMR (400 MHz, DMSO- $d_{6}$ ): $\delta=2.55$ (s, $3 \mathrm{H} \mathrm{CH}_{3}-\mathrm{S}$ ); 3.31 (s, $3 \mathrm{H}, \mathrm{CH}_{3}-\mathrm{N}$ ); 8.24 (s, $1 \mathrm{H}, \mathrm{NH}_{2}$ ); 8.91 (s, $1 \mathrm{H}, \mathrm{NH}_{2}$ ); 9.90 (s, $1 \mathrm{H}, \mathrm{CHO}) .{ }^{13} \mathrm{C}$ NMR $\left(100 \mathrm{MHz}, \mathrm{DMSO}-d_{6}\right): \delta=14.48\left(\mathrm{~S}-\mathrm{CH}_{3}\right) ; 28.98\left(\mathrm{~N}_{-} \mathrm{CH}_{3}\right) ; 94.33$ $\left(\mathrm{C}_{5}\right) ; 167.78\left(\mathrm{C}_{2}\right) ; 160.06\left(\mathrm{C}_{6}\right) ; 162.06\left(\mathrm{C}_{4}\right) ; 188.52(\mathrm{CHO}) . \mathrm{IR}(\mathrm{KBr}) \mathrm{cm}^{-1}$ 3375, $3272\left(\mathrm{NH}_{2} s t\right)$, 1630, 1605 (C=O st). MS (70 eV) m/z (\%) 199 (M+24), 171 (100), 68 (62), 57 (64). HR-MS calc. for $\mathrm{C}_{7} \mathrm{H}_{9} \mathrm{~N}_{3} \mathrm{O}_{2} \mathrm{~S} 199.0415$ found 199.0427 .

4-Amino-2-methoxy-1-methyl-6-oxo-1,6-dihydropyr-imidine-5-carbaldehyde 5b. $0.155 \mathrm{~g}$ of 6-Amino-2-methoxy-3-methylpyrimidin-4(3H)-one reacts accorging to the general procedure $\mathrm{B}$ for 4 hours in the ice bath. White solid. Yield 70\%, mp 218-220 ${ }^{\circ} \mathrm{C}$. ${ }^{1} \mathrm{H}$ NMR (400 MHz, DMSO$\left.d_{6}\right): \delta=3.20$ (s, 3H, $\mathrm{CH}_{3}-\mathrm{N}$ ); 4.00 (s, 3H, $\mathrm{CH}_{3}-\mathrm{O}$ ); 7.94 (s, $1 \mathrm{H}, \mathrm{NH}_{2}$ ); 8.95 (s, $1 \mathrm{H}, \mathrm{NH}_{2}$ ); 9.86 (s, $1 \mathrm{H}, \mathrm{CHO}) .{ }^{13} \mathrm{C}$ NMR (100 MHz, DMSO-d $): \delta=26.22\left(\mathrm{~N}-\mathrm{CH}_{3}\right) ; 55.59\left(\mathrm{O}_{-} \mathrm{CH}_{3}\right) ; 93.61\left(\mathrm{C}_{5}\right)$; $158.26\left(\mathrm{C}_{2}\right) ; 162.20\left(\mathrm{C}_{6}\right) ; 162.84\left(\mathrm{C}_{4}\right) ; 187.28(\mathrm{CHO}) . \mathrm{IR}(\mathrm{KBr}) \mathrm{cm}^{-1} 3193(\mathrm{NH} s t), 1733(\mathrm{C}=\mathrm{O}$ $s t)$. MS (70 eV) m/z (\%) $183\left(\mathrm{M}^{+}, 31\right), 155$ (100), 125 (21), 42 (23). HR-MS calc. for $\mathrm{C}_{7} \mathrm{H}_{9} \mathrm{~N}_{3} \mathrm{O}_{3}$ 183.0644 found 183.0665 .

$\boldsymbol{N}$-(5-Formyl-1-methyl-2,6-dioxo-1,2,3,6-tetrahydropyrimidin-4-yl)formamide 6g. $0.155 \mathrm{~g}$ of 6-Amino-2-methoxy-3-methylpyrimidin-4(3H)-one in $1.5 \mathrm{~mL}$ of DMF were added to Vilsmeier's reagent $\left(0.15 \mathrm{~mL}\right.$ of $\mathrm{POCl}_{3}$ in $0.5 \mathrm{~mL}$ of DMF) and stirred for 2 hours at $50{ }^{\circ} \mathrm{C}$. The 
solution was cooled to room temperature and then poured into crushed ice. The solid obtained was filtered and washed with water. White solid. Yield $86 \%$, mp $>300{ }^{\circ} \mathrm{C}\left(248-252{ }^{\circ} \mathrm{C}\right.$, decomposed). ${ }^{1} \mathrm{H}$ NMR (400 MHz, DMSO- $d_{6}$ ): $\delta=3.17$ (s, 3H, $\mathrm{CH}_{3}-\mathrm{N}$ ); 11.89 (s, $1 \mathrm{H}, \mathrm{NH}$ ); 9.62 (s, $1 \mathrm{H}, \mathrm{NH}) ; 8.92$ (s, $1 \mathrm{H}, \mathrm{NH}-\mathrm{CHO}) ; 9.82(\mathrm{~s}, 1 \mathrm{H}, \mathrm{CHO}) .{ }^{13} \mathrm{C}$ NMR $\left(100 \mathrm{MHz}, \mathrm{DMSO}-d_{6}\right): \delta$ $=26.06\left(\mathrm{~N}-\mathrm{CH}_{3}\right) ; 92.34\left(\mathrm{C}_{5}\right) ; 162.04\left(\mathrm{C}_{2}\right) ; 148.61\left(\mathrm{C}_{6}\right) ; 151.48\left(\mathrm{C}_{4}\right) ; 163.61(\mathrm{NH}-\mathrm{CHO}) ; 188.53$ (CHO). IR (KBr) cm $3181(\mathrm{NH} s t), 1663(\mathrm{C}=\mathrm{O} s t), 1524(\mathrm{NH} \delta)$. MS (70 eV) $m / z(\%) 197\left(\mathrm{M}^{+}\right.$, 30), 169 (26), 141 (100), 111 (61), 68 (83). HR-MS calc. for $\mathrm{C}_{7} \mathrm{H}_{7} \mathrm{~N}_{3} \mathrm{O}_{4} 197.0437$ found 197.0450.

6-Amino-3-methyl-2,4-dioxo-1,2,3,4-tetrahydropyrimidine-5-carbaldehyde $3 g$. 0.197 g (1 $\mathrm{mmol})$ of $\mathbf{6 g}$ were heated to boiled in $30 \mathrm{~mL}$ of aqueous $\mathrm{NaOH}(0.4 \mathrm{M})$ for 1 hour. The reaction was left to cool at room temperature and the white solid was filtered. The solid recrystallized from ethanol. Yield $84 \%$, m.p. $>300^{\circ} \mathrm{C}$ (bibliographic mp $>300^{\circ} \mathrm{C}$ )..$^{17}$

\section{General procedure for the synthesis of pyrido[2,3- $d$ ]pyrimidines}

6-Aminopyrimidin-5-carboxaldehyde $\mathbf{1}, \mathbf{5 a}$ and $\mathbf{5 b}$ reacts with equimolecular amounts of acetophenones 7 with catalytic amount of $\mathrm{BF}_{3}-\mathrm{Et}_{2} \mathrm{O}$; the mixture of reaction was heated in bath oil at $160{ }^{\circ} \mathrm{C}$ for 30 seconds. The resulting dark brown solution was diluted in ethanol and cooled to room temperature. The resulting precipitated was filtered, washed with ethanol and recrystallized to afford the products $\mathbf{8 , 9}$ and $\mathbf{1 0}$ respectively.

1,3-Dimethyl-7-phenylpyrido[2,3-d]pyrimidine-2,4(1H, 3H)-dione 8a. Yellow solid, recrystallized from ethanol, yield $65 \%$, mp 157-159 ${ }^{\circ} \mathrm{C} .{ }^{1} \mathrm{H}$ NMR $\left(400 \mathrm{MHz}, \mathrm{CDCl}_{3}\right): \delta=3.49$ (s, $3 \mathrm{H}, \mathrm{N}_{1}-\mathrm{CH}_{3}$ ), $3.82\left(\mathrm{~s}, 3 \mathrm{H}, \mathrm{N}_{3}-\mathrm{CH}_{3}\right), 7.51-7.5\left(\mathrm{~m}, 3 \mathrm{H}, \mathrm{H}_{m}\right.$ and $\left.\mathrm{H}_{p}\right), 7.66\left(\mathrm{~d}, 1 \mathrm{H}, \mathrm{H}_{6}, J=8.03\right.$ $\mathrm{Hz}$ ), 8.13-8.10 (m, 2H, $\mathrm{H}_{\mathrm{o}}$ ), 8.49 (d, $\left.1 \mathrm{H}, \mathrm{H}_{5}, J=8.03 \mathrm{~Hz}\right) .{ }^{13} \mathrm{C} \mathrm{NMR}\left(100 \mathrm{MHz}, \mathrm{CDCl}_{3}\right): \delta=$ $28.4\left(\mathrm{~N}_{1}-\mathrm{CH}_{3}\right), 29.4\left(\mathrm{~N}_{3}-\mathrm{CH}_{3}\right), 109.3\left(\mathrm{C}_{4 \mathrm{a}}\right), 115.5\left(\mathrm{C}_{6}\right), 127.8\left(\mathrm{C}_{o}\right), 129.3\left(\mathrm{C}_{m}\right), 131.0\left(\mathrm{C}_{p}\right), 137.8$ $\left(\mathrm{C}_{i}\right), 138.6\left(\mathrm{C}_{7}\right), 138.7\left(\mathrm{C}_{5}\right), 151.1\left(\mathrm{C}_{4}\right), 152.0\left(\mathrm{C}_{2}\right), 161.6\left(\mathrm{C}_{8 \mathrm{a}}\right)$. IR $(\mathrm{KBr}) \mathrm{cm}^{-1}$ 1706, 1658, $(\mathrm{C}=\mathrm{O}$ $s t)$. MS (70 eV) m/z (\%) 267 (M 267.1008 found 267.1010. Anal. Calcd for $\mathrm{C}_{15} \mathrm{H}_{13} \mathrm{~N}_{3} \mathrm{O}_{2}$ : C, 67.40; H, 4.90; N, 15.72. Found: C, 67.70; H, 4.86; N, 15.26.

1,3-Dimethyl-7-(4-methylphenyl)pyrido[2,3-d]pyrimidine-2,4(1H, 3H)-dione 8b. Yellow crystalline solid, recrystallized from ethanol and DMF mixture, yield $50 \%$, mp $175-177^{\circ} \mathrm{C} .{ }^{1} \mathrm{H}$ NMR (400 MHz, $\mathrm{CDCl}_{3}$ ): $\delta=2.44$ (s, 3H, $\mathrm{CH}_{3}$ ), 3.49 (s, 3H, $\mathrm{N}_{1}-\mathrm{CH}_{3}$ ), 3.80 (s, $3 \mathrm{H}, \mathrm{N}_{3}-\mathrm{CH}_{3}$ ), 7.31 (d, $2 \mathrm{H}, \mathrm{H}_{o}, J=8.54 \mathrm{~Hz}$ ), 7.61 (d, $1 \mathrm{H}, \mathrm{H}_{6}, J=8.03 \mathrm{~Hz}$ ), 8.00 (d, $2 \mathrm{H}, \mathrm{H}_{m}, J=8.28 \mathrm{~Hz}$ ), 8.44 (d, $\left.1 \mathrm{H}, \mathrm{H}_{5}, J=8.28 \mathrm{~Hz}\right) .{ }^{13} \mathrm{C}$ NMR $\left(100 \mathrm{MHz}, \mathrm{CDCl}_{3}\right): \delta=21.3\left(\mathrm{~N}_{1}-\mathrm{CH}_{3}\right), 28.3\left(\mathrm{~N}_{3}-\mathrm{CH}_{3}\right), 29.3$ $\left(\mathrm{CH}_{3}\right), 108.8\left(\mathrm{C}_{4 \mathrm{a}}\right), 114.7\left(\mathrm{C}_{6}\right), 127.4\left(\mathrm{C}_{m}\right), 129.6\left(\mathrm{C}_{o}\right), 134.9\left(\mathrm{C}_{i}\right), 138.2\left(\mathrm{C}_{5}\right), 141.1\left(\mathrm{C}_{p}\right), 150.8$ $\left(\mathrm{C}_{4}\right), 151.7\left(\mathrm{C}_{2}\right), 161.3\left(\mathrm{C}_{8 \mathrm{a}}\right)$. IR $(\mathrm{KBr}) \mathrm{cm}^{-1}$ 1704, 1662, (C=O st). MS (70 eV) m/z (\%) $281\left(\mathrm{M}^{+}\right.$, 100), 252 (51), 169 (50). HR-MS calc. for $\mathrm{C}_{16} \mathrm{H}_{15} \mathrm{~N}_{3} \mathrm{O}_{2} 281.1164$ found 281.1159. Anal. Calcd for $\mathrm{C}_{16} \mathrm{H}_{15} \mathrm{~N}_{3} \mathrm{O}_{2}$ : C, 68.31; H, 5.37; N, 14.94. Found: C, 67.90; H, 4.96; N, 14.76.

7-(4-Methoxyphenyl)-1,3-dimethylpyrido[2,3-d]pyrimidine-2,4(1H, 3H)-dione 8c. White solid, recrystallized from ethanol and DMF mixture, yield 60\%, mp $163-165{ }^{\circ} \mathrm{C} .{ }^{1} \mathrm{H}$ NMR (400 $\mathrm{MHz}, \mathrm{CDCl}_{3}$ ): $\delta=3.47$ (s, 3H, N $\mathrm{N}_{1}-\mathrm{CH}_{3}$ ), 3.79 (s, 3H, N $\mathrm{N}_{3}-\mathrm{CH}_{3}$ ), 3.88 (s, 3H, $\left.\mathrm{OCH}_{3}\right), 7.00(\mathrm{~d}, 2 \mathrm{H}$, 
$\mathrm{H}_{0}, J=8.78 \mathrm{~Hz}$ ), 7.56 (d, $\left.1 \mathrm{H}, \mathrm{H}_{6}, J=8.28 \mathrm{~Hz}\right), 8.07$ (d, $\left.2 \mathrm{H}, \mathrm{H}_{\mathrm{m}}, J=8.78 \mathrm{~Hz}\right), 8.41$ (d, $1 \mathrm{H}, \mathrm{H}_{5}, J$ $=8.03 \mathrm{~Hz}) .{ }^{13} \mathrm{C}$ NMR $\left(100 \mathrm{MHz}, \mathrm{CDCl}_{3}\right): \delta=28.6\left(\mathrm{~N}_{1}-\mathrm{CH}_{3}\right), 29.6\left(\mathrm{~N}_{3}-\mathrm{CH}_{3}\right), 55.4\left(\mathrm{OCH}_{3}\right)$, $108.5\left(\mathrm{C}_{4 \mathrm{a}}\right), 114.3\left(\mathrm{C}_{6}\right), 114.3\left(\mathrm{C}_{m}\right), 129.3\left(\mathrm{C}_{o}\right), 130.3\left(\mathrm{C}_{i}\right), 138.4\left(\mathrm{C}_{5}\right), 151.0\left(\mathrm{C}_{4}\right), 152.0\left(\mathrm{C}_{2}\right)$, $161.1\left(\mathrm{C}_{7}\right), 161.7\left(\mathrm{C}_{8 \mathrm{a}}\right), 162.2\left(\mathrm{C}_{p}\right)$. IR $(\mathrm{KBr}) \mathrm{cm}^{-1}$ 1702, 1661, $(\mathrm{C}=\mathrm{O} s t)$. MS $(70 \mathrm{eV}) \mathrm{m} / z(\%)=$ 297 ( $\mathrm{M}^{+}, 100$ ), 268 (35), 185 (31). HR-MS calc. for $\mathrm{C}_{16} \mathrm{H}_{15} \mathrm{~N}_{3} \mathrm{O}_{3} 297.1113$ found 297.1114. Anal. Calcd for $\mathrm{C}_{16} \mathrm{H}_{15} \mathrm{~N}_{3} \mathrm{O}_{3}$ : C, 64.64; H, 5.09; N, 14.13. Found: C, 64.26; H, 4.86; N, 14.52.

1,3-Dimethyl-7-(3,4,5-trimethoxyphenyl)pyrido[2,3-d]pyrimidine-2,4(1H, 3H)-dione 8d. Yellow crystalline solid, recrystallized from ethanol and DMF mixture, yield 40\%, mp 211- 213 ${ }^{\circ} \mathrm{C} .{ }^{1} \mathrm{H}$ NMR (400 MHz, $\mathrm{CDCl}_{3}$ ): $\delta=3.49$ (s, 3H, N $\mathrm{N}_{1}-\mathrm{CH}_{3}$ ), 3.81 (s, 3H, $\mathrm{N}_{3}-\mathrm{CH}_{3}$ ), 3.93 (s, 3H, $\mathrm{C}_{p}-\mathrm{OCH}_{3}$ ), 3.98 (s, 3H, $\mathrm{C}_{m}-\mathrm{OCH}_{3}$ ), 7.37 (s, $2 \mathrm{H}, \mathrm{H}_{o}$ ), 7.60 (d, $\left.1 \mathrm{H}, \mathrm{H}_{6}, J=8.28 \mathrm{~Hz}\right), 8.47$ (d, $1 \mathrm{H}$, $\left.\mathrm{H}_{5}, J=8.28 \mathrm{~Hz}\right) .{ }^{13} \mathrm{C}$ NMR $\left(100 \mathrm{MHz}, \mathrm{CDCl}_{3}\right): \delta=28.4\left(\mathrm{~N}_{1}-\mathrm{CH}_{3}\right), 29.3\left(\mathrm{~N}_{3}-\mathrm{CH}_{3}\right), 56.6\left(\mathrm{C}_{m^{-}}\right.$ $\left.\mathrm{OCH}_{3}\right), 61.3\left(\mathrm{C}_{p}-\mathrm{OCH}_{3}\right), 105.2\left(\mathrm{C}_{o}\right), 109.1\left(\mathrm{C}_{4 \mathrm{a}}\right), 115.2\left(\mathrm{C}_{6}\right), 133.2\left(\mathrm{C}_{i}\right), 138.5\left(\mathrm{C}_{5}\right), 141.0\left(\mathrm{C}_{p}\right)$, $150.9\left(\mathrm{C}_{4}\right), 151.9\left(\mathrm{C}_{2}\right), 153.9\left(\mathrm{C}_{m}\right), 161.1\left(\mathrm{C}_{7}\right), 161.6\left(\mathrm{C}_{8 \mathrm{a}}\right)$. IR $(\mathrm{KBr}) \mathrm{cm}^{-1}$ 1710, 1655, (C=O st). MS (70 eV) m/z (\%)357 (M+100), 342 (47), 314 (25). HR-MS calc. for $\mathrm{C}_{18} \mathrm{H}_{19} \mathrm{~N}_{3} \mathrm{O}_{5} 357.1325$ found 357.1315. Anal. Calcd for $\mathrm{C}_{18} \mathrm{H}_{19} \mathrm{~N}_{3} \mathrm{O}_{5}$ : C, 60.50; H, 5.36; N, 11.76. Found: C, 60.36; H, 5.86; N, 11.92 .

7-(1,1'-Biphenyl-4yl)-1,3-dimethylpyrido[2,3-d]pyrimidine-2,4(1H, ,3H)-dione 8e. Yellow solid, purified by column chromatography using chloroform as eluant, yield 60\%, mp 233-235 ${ }^{\circ} \mathrm{C} .{ }^{1} \mathrm{H}$ NMR $\left(400 \mathrm{MHz}, \mathrm{CDCl}_{3}\right): \delta=3.49$ (s, 3H, N $\mathrm{N}_{1}-\mathrm{CH}_{3}$ ), 3.83 (s, 3H, $\left.\mathrm{N}_{3}-\mathrm{CH}_{3}\right), 7.40(\mathrm{t}, 1 \mathrm{H}$, $\mathrm{H}_{p}$ ), 7.45 (t, $2 \mathrm{H}, \mathrm{H}_{m}$ ), 7.65 (d, 2H, $\mathrm{H}_{o}, J=8.28 \mathrm{~Hz}$ ), 7.68 (d, $\left.1 \mathrm{H}, \mathrm{H}_{6}, J=8.03 \mathrm{~Hz}\right), 7.73$ (d, 2H, $\left.\mathrm{H}_{m}, J=8.79 \mathrm{~Hz}\right), 8.19$ (d, $\left.2 \mathrm{H}, \mathrm{H}_{o}, J=8.54 \mathrm{~Hz}\right), 8.49$ (d, $\left.1 \mathrm{H}, \mathrm{H}_{5}, J=8.03 \mathrm{~Hz}\right) .{ }^{13} \mathrm{C}$ NMR $(100$ $\left.\mathrm{MHz}, \mathrm{CDCl}_{3}\right): \delta=28.4\left(\mathrm{~N}_{1}-\mathrm{CH}_{3}\right), 29.4\left(\mathrm{~N}_{3}-\mathrm{CH}_{3}\right), 109.0\left(\mathrm{C}_{4 \mathrm{a}}\right), 115.0\left(\mathrm{C}_{6}\right), 127.1\left(\mathrm{C}_{o}\right), 127.6$ $\left(\mathrm{C}_{m}\right), 127.9\left(\mathrm{C}_{o}\right), 128.9\left(\mathrm{C}_{m}\right), 136.8\left(\mathrm{C}_{i}\right), 138.3\left(\mathrm{C}_{5}\right), 150.7\left(\mathrm{C}_{4}\right), 151.7\left(\mathrm{C}_{2}\right), 160.9\left(\mathrm{C}_{7}\right), 161.3$ $\left(\mathrm{C}_{8 \mathrm{a}}\right)$. IR $(\mathrm{KBr}) \mathrm{cm}^{-1}$ 1708, 1656, $(\mathrm{C}=\mathrm{O} s t)$. MS (70 eV) m/z (\%) = $343\left(\mathrm{M}^{+}, 100\right), 314$ (32), 231 (30). HR-MS calc. for $\mathrm{C}_{21} \mathrm{H}_{17} \mathrm{~N}_{3} \mathrm{O}_{2} 343.1321$ found 343.1313. Anal. Calcd for $\mathrm{C}_{21} \mathrm{H}_{17} \mathrm{~N}_{3} \mathrm{O}_{2}$ : C, 73.45; H, 4.99; N, 12.24. Found: C, 69.96; H, 5.12; N, 11.96.

7-(4-Hydroxyphenyl)-1,3-dimethylpyrido[2,3-d]pyrim-idine-2,4(1H,3H)-dione 8f. Yellow solid, the residue was washed with hot hexanes, yield $40 \%$, mp $>300{ }^{\circ} \mathrm{C} .{ }^{1} \mathrm{H}$ NMR (400 MHz, $\mathrm{CDCl}_{3}$ ): $\delta=3.25$ (s, 3H, N $\mathrm{N}_{1}-\mathrm{CH}_{3}$ ), 3.59 (s, 3H, N $\mathrm{N}_{3}-\mathrm{CH}_{3}$ ), 6.87 (d, 2H, $\mathrm{H}_{m}, J=8.53 \mathrm{~Hz}$ ), 7.71 (d, $\left.1 \mathrm{H}, \mathrm{H}_{6}, J=8.28 \mathrm{~Hz}\right), 8.03$ (d, $\left.2 \mathrm{H}, \mathrm{CH}_{o}, J=8.54 \mathrm{~Hz}\right), 8.24$ (d, $\left.1 \mathrm{H}, \mathrm{H}_{5}, J=8.28 \mathrm{~Hz}\right) .{ }^{13} \mathrm{C} \mathrm{NMR}$ $\left(100 \mathrm{MHz}, \mathrm{CDCl}_{3}\right): \delta=28.3\left(\mathrm{~N}_{1}-\mathrm{CH}_{3}\right), 29.3\left(\mathrm{~N}_{3}-\mathrm{CH}_{3}\right), 107.3\left(\mathrm{C}_{4 \mathrm{a}}\right), 114.4\left(\mathrm{C}_{6}\right), 116.4\left(\mathrm{C}_{m}\right), 127.8$ $\left(\mathrm{C}_{i}\right), 129.5\left(\mathrm{C}_{o}\right), 138.1\left(\mathrm{C}_{5}\right), 150.1\left(\mathrm{C}_{4}\right), 150.8\left(\mathrm{C}_{2}\right), 159.7\left(\mathrm{C}_{p}\right), 159.9\left(\mathrm{C}_{7}\right), 160.2\left(\mathrm{C}_{8 \mathrm{a}}\right) . \mathrm{IR}(\mathrm{KBr})$ $\mathrm{cm}^{-1} 3179$ (OH st), 1699, 1633, (C=O st). MS (70 eV) m/z (\%)283 (M+1 100), 254 (45), 171 (47). HR-MS calc, for $\mathrm{C}_{15} \mathrm{H}_{13} \mathrm{~N}_{3} \mathrm{O}_{3}$ 283.0957, found 283.0965. Anal. Calcd for $\mathrm{C}_{15} \mathrm{H}_{13} \mathrm{~N}_{3} \mathrm{O}_{3}$ : C, 63.60; H, 4.63; N, 14.83. Found: C, 63.86; H, 4.26; N, 14.82 .

1,3-Dimethyl-7-(4-nitrophenyl)pyrido[2,3-d]pyrimidine-2,4(1H, 3H)-dione 8g. Yellow solid, recrystallized from DMF, yield 50\%, mp 283-285 ${ }^{\circ} \mathrm{C} .{ }^{1} \mathrm{H}$ NMR (400 MHz, $\mathrm{CDCl}_{3}$ ): $\delta=3.51$ (s, $3 \mathrm{H}, \mathrm{N}_{1}-\mathrm{CH}_{3}$ ), 3.83 (s, $\left.3 \mathrm{H}, \mathrm{N}_{3}-\mathrm{CH}_{3}\right), 7.71\left(\mathrm{~d}, 1 \mathrm{H}, \mathrm{H}_{6}, J=8.03 \mathrm{~Hz}\right.$ ), $8.37-8.26$ (dd, $4 \mathrm{H}, \mathrm{H}_{\text {arom }}, J=$ $9.03 \mathrm{~Hz}$ ), 8.58 (d, $\left.1 \mathrm{H}, \mathrm{H}_{5}, J=8.03 \mathrm{~Hz}\right) .{ }^{13} \mathrm{C} \mathrm{NMR}\left(100 \mathrm{MHz}, \mathrm{CDCl}_{3}\right): \delta=28.4\left(\mathrm{~N}_{1}-\mathrm{CH}_{3}\right), 29.5$ $\left(\mathrm{N}_{3}-\mathrm{CH}_{3}\right), 110.4\left(\mathrm{C}_{4 \mathrm{a}}\right), 115.7\left(\mathrm{C}_{6}\right), 124.1\left(\mathrm{C}_{m}\right), 128.3\left(\mathrm{C}_{o}\right), 139.0\left(\mathrm{C}_{5}\right), 143.4\left(\mathrm{C}_{i}\right), 149.2\left(\mathrm{C}_{p}\right)$, 
$151.2\left(\mathrm{C}_{4}\right), 151.5\left(\mathrm{C}_{2}\right), 158.7\left(\mathrm{C}_{7}\right), 161.0\left(\mathrm{C}_{8 \mathrm{a}}\right) . \mathrm{IR}(\mathrm{KBr}) \mathrm{cm}^{-1}$ 1705, 1660, (C=O st). MS (70 eV) $m / z(\%)=312\left(\mathrm{M}^{+}, 13\right), 200$ (8), 28 (43), 18 (100). HR-MS calc. for $\mathrm{C}_{15} \mathrm{H}_{12} \mathrm{~N}_{4} \mathrm{O}_{4} 312.0859$ found 312.0852. Anal. Calcd for $\mathrm{C}_{15} \mathrm{H}_{12} \mathrm{~N}_{4} \mathrm{O}_{4}$ : C, 57.69; H, 3.87; N, 17.94. Found: C, 56.92; H, 4.16; N, 16.88 .

7-(4-Chlorophenyl)-1,3-dimethylpyrido[2,3-d]pyrimid-ine-2,4(1H, 3H)-dione 8h. Yellow solid, recrystallized from ethanol and DMF mixture, yield 40\%, mp 138-140 ${ }^{\circ} \mathrm{C}$. ${ }^{1} \mathrm{H}$ NMR (400 $\mathrm{MHz}, \mathrm{CDCl}_{3}$ ): $\delta=3.48$ (s, 3H, N $\mathrm{N}_{1}-\mathrm{CH}_{3}$ ), 3.79 (s, 3H, $\mathrm{N}_{3}-\mathrm{CH}_{3}$ ), 7.46 (d, $2 \mathrm{H}, \mathrm{H}_{o}, J=9.04 \mathrm{~Hz}$ ), 7.60 (d, $\left.1 \mathrm{H}, \mathrm{H}_{6}, J=8.03 \mathrm{~Hz}\right), 8.04$ (d, $\left.2 \mathrm{H}, \mathrm{H}_{m}, J=8.78 \mathrm{~Hz}\right), 8.48$ (d, $\left.1 \mathrm{H}, \mathrm{H}_{5}, J=8.28 \mathrm{~Hz}\right) .{ }^{13} \mathrm{C}$ NMR (100 MHz, CDCl $): \delta=28.8\left(\mathrm{~N}_{1}-\mathrm{CH}_{3}\right), 29.7\left(\mathrm{~N}_{3}-\mathrm{CH}_{3}\right), 109.5\left(\mathrm{C}_{4 \mathrm{a}}\right), 115.2\left(\mathrm{C}_{6}\right), 128.7\left(\mathrm{C}_{m}\right)$, $129.2\left(\mathrm{C}_{o}\right), 136.2\left(\mathrm{C}_{i}\right), 137.3\left(\mathrm{C}_{p}\right), 138.5\left(\mathrm{C}_{5}\right), 151.1\left(\mathrm{C}_{4}\right), 151.9\left(\mathrm{C}_{2}\right), 160.2\left(\mathrm{C}_{7}\right), 161.5\left(\mathrm{C}_{8 \mathrm{a}}\right) . \mathrm{IR}$ $(\mathrm{KBr}) \mathrm{cm}^{-1}$ 1705, 1658, (C=O st). MS (70 eV) $m / z(\%)$ 303/301 ( $\left.\mathrm{M}^{+2} / \mathrm{M}^{+}, 35 / 100\right), 273$ (17). HRMS calc. for $\mathrm{C}_{15} \mathrm{H}_{12} \mathrm{ClN}_{3} \mathrm{O}_{2} 301.0618$ found 301.0616. Anal. Calcd for $\mathrm{C}_{15} \mathrm{H}_{12} \mathrm{ClN}_{3} \mathrm{O}_{2}$ : C, 59.71; H, 4.01; N, 13.93. Found: C, 58.92; H, 3.96; N, 13.88.

7-(4-Bromophenyl)-1,3-dimethylpyrido[2,3-d]pyrimidine-2,4(1H, 3H)-dione 8i. White solid, recrystallized from ethanol and DMF mixture, yield 40\%, mp 217.5-219.0 ${ }^{\circ} \mathrm{C} .{ }^{1} \mathrm{H}$ NMR (400 $\mathrm{MHz}, \mathrm{CDCl}_{3}$ ): $\delta=3.48$ (s, 3H, N $\mathrm{N}_{1} \mathrm{CH}_{3}$ ), 3.79 (s, 3H, N $\mathrm{N}_{3}-\mathrm{CH}_{3}$ ), $7.64-7.60$ (t, 3H, $\mathrm{H}_{o}$ and $\mathrm{H}_{6}$ ), 7.97 (d, $\left.2 \mathrm{H}, \mathrm{H}_{m}, J=8.78 \mathrm{~Hz}\right), 8.48\left(\mathrm{~d}, 1 \mathrm{H}, \mathrm{H}_{5}, J=8.28 \mathrm{~Hz}\right) .{ }^{13} \mathrm{C}$ NMR $\left(100 \mathrm{MHz}, \mathrm{CDCl}_{3}\right): \delta=$ $28.5\left(\mathrm{~N}_{1}-\mathrm{CH}_{3}\right), 29.4\left(\mathrm{~N}_{3}-\mathrm{CH}_{3}\right), 109.3\left(\mathrm{C}_{4 \mathrm{a}}\right), 114.9\left(\mathrm{C}_{6}\right), 125.4\left(\mathrm{C}_{i}\right), 129.0\left(\mathrm{C}_{m}\right), 132.2\left(\mathrm{C}_{o}\right), 136.1$ $\left(\mathrm{C}_{p}\right), 138.6\left(\mathrm{C}_{5}\right), 150.2\left(\mathrm{C}_{4}\right), 151.6\left(\mathrm{C}_{2}\right), 160.0\left(\mathrm{C}_{7}\right), 161.2\left(\mathrm{C}_{8 \mathrm{a}}\right) . \mathrm{IR}(\mathrm{KBr}) \mathrm{cm}^{-1}$ 1705, 1656, $(\mathrm{C}=\mathrm{O}$ $s t)$. MS (70 eV) m/z (\%)347/345 (M $\left.\mathrm{M}^{+2} / \mathrm{M}^{+}, 93 / 100\right), 318 / 316$ (36/34), 234/232 (35/38), 154 (32). HR-MS calc. for $\mathrm{C}_{15} \mathrm{H}_{12} \mathrm{~N}_{3} \mathrm{O}_{2} \mathrm{Br}$ 345.0113, found 345.0118. Anal. Calcd for $\mathrm{C}_{15} \mathrm{H}_{12} \mathrm{~N}_{3} \mathrm{O}_{2} \mathrm{Br}$ : C, 52.04; H, 3.49; N, 12.14. Found: C, 52.22; H, 3.52; N, 12.48.

7-(4-Fluorophenyl)-1,3-dimethylpyrido[2,3-d]pyrimidine-2,4(1H, 3H)-dione 8j. White solid, recrystallized from ethanol and DMF mixture, yield 60\%, mp 207-209 ${ }^{\circ} \mathrm{C}$. ${ }^{1} \mathrm{H}$ NMR (400 MHz, $\left.\mathrm{CDCl}_{3}\right): \delta=3.48$ (s, 3H, N $\mathrm{CH}_{3}$ ), 3.80 (s, 3H, N $\mathrm{N}_{3} \mathrm{CH}_{3}$ ), 7.18 (t, 2H, $\mathrm{H}_{m}$ ), 7.59 (d, $1 \mathrm{H}, \mathrm{H}_{6}, J=$ $8.03 \mathrm{~Hz}), 8.11\left(\mathrm{~m}, 2 \mathrm{H}, \mathrm{H}_{o}\right), 8.48\left(\mathrm{~d}, 1 \mathrm{H}, \mathrm{H}_{5}, J=8.03 \mathrm{~Hz}\right) .{ }^{13} \mathrm{C} \mathrm{NMR}\left(100 \mathrm{MHz}, \mathrm{CDCl}_{3}\right): \delta=28.4$ $\left(\mathrm{N}_{1}-\mathrm{CH}_{3}\right), 29.4\left(\mathrm{~N}_{3}-\mathrm{CH}_{3}\right), 108.9\left(\mathrm{C}_{4 \mathrm{a}}\right), 114.8\left(\mathrm{C}_{6}\right), 116.1\left(\mathrm{C}_{m}\right), 129.5\left(\mathrm{C}_{o}\right), 138.5\left(\mathrm{C}_{5}\right), 150.7\left(\mathrm{C}_{4}\right)$, $151.6\left(\mathrm{C}_{2}\right), 160.1\left(\mathrm{C}_{7}\right), 161.3\left(\mathrm{C}_{8 \mathrm{a}}\right), 163.2\left(\mathrm{C}_{i}\right), 165.7\left(\mathrm{C}_{p}\right) . \mathrm{IR}(\mathrm{KBr}) \mathrm{cm}^{-1}$ 1710, 1668, (C=O st). MS (70 eV) m/z (\%) 285 (M $\left.{ }^{+}, 100\right), 257$ (43), 173 (54). HR-MS calc. for $\mathrm{C}_{15} \mathrm{H}_{12} \mathrm{~N}_{3} \mathrm{O}_{2} \mathrm{~F} 285.0914$ found 285.0913. Anal. Calcd for $\mathrm{C}_{15} \mathrm{H}_{12} \mathrm{~N}_{3} \mathrm{O}_{2} \mathrm{~F}$ : C, 63.15; $\mathrm{H}, 4.24 ; \mathrm{N}, 14.73$. Found: C, 62.92; $\mathrm{H}$, 4.46; N, 14.38 .

1,3-Dimethyl-7-(2-oxo-2H-chromen-3-yl)pyrido[2,3-d]pyrimidine-2,4(1H, 3H)-dione $8 \mathrm{k}$. Brown solid, recrystallized from DMF, yield 60\%, mp 284.5-286.0 ${ }^{\circ} \mathrm{C}{ }^{1} \mathrm{H}$ NMR (400 MHz, $\left.\mathrm{CDCl}_{3}\right): \delta=3.51\left(\mathrm{~s}, 3 \mathrm{H}, \mathrm{N}_{1}-\mathrm{CH}_{3}\right), 3.82\left(\mathrm{~s}, 3 \mathrm{H}, \mathrm{N}_{3}-\mathrm{CH}_{3}\right), 7.38-7.33\left(\mathrm{~m}, 2 \mathrm{H}, \mathrm{H}_{\text {aryl }}\right), 7.63(\mathrm{t}, 1 \mathrm{H}$, $\mathrm{H}_{\text {aryl }}$ ), 7.71 (d, $1 \mathrm{H}, \mathrm{H}_{\text {aryl }}, J=7.78 \mathrm{~Hz}$ ), $8.52-8.41$ (dd, 2H, $\mathrm{H}_{5}$ and $\mathrm{H}_{6}, J=8.03 \mathrm{~Hz}$ ), 8.85 (s, 1H, $\mathrm{CH}) .{ }^{13} \mathrm{C}$ NMR (100 MHz, $\left.\mathrm{CDCl}_{3}\right): \delta=28.1\left(\mathrm{~N}_{1}-\mathrm{CH}_{3}\right), 29.1\left(\mathrm{~N}_{3}-\mathrm{CH}_{3}\right), 110.0\left(\mathrm{C}_{4}\right), 116.2\left(\mathrm{C}_{\text {aryl }}\right)$, $118.6\left(\mathrm{C}_{6}\right), 118.7\left(\mathrm{C}_{q}\right), 123.5\left(\mathrm{C}_{\text {q-aryl }}\right), 124.8\left(\mathrm{CH}_{\text {aryl }}\right), 129.0\left(\mathrm{CH}_{\text {aryl }}\right), 133.0\left(\mathrm{CH}_{\text {aryl }}\right), 138.6\left(\mathrm{C}_{5}\right)$, $144.4(\mathrm{CH}), 150.0\left(\mathrm{C}_{7}\right), 151.2\left(\mathrm{C}_{2}\right), 154.0\left(\mathrm{C}_{4}\right), 154.8\left(\mathrm{C}_{\text {q-aryl }}\right), 159.3(\mathrm{C}=\mathrm{O}), 160.7\left(\mathrm{C}_{8 \mathrm{a}}\right) . \mathrm{IR}$ $(\mathrm{KBr}) \mathrm{cm}^{-1}$ 1714, 1660, (C=O st). MS (70 eV) m/z (\%) = $335\left(\mathrm{M}^{+}, 100\right), 306$ (47), 223 (56), 195 
(20). HR-MS calc. For $\mathrm{C}_{18} \mathrm{H}_{13} \mathrm{~N}_{3} \mathrm{O}_{4} 335.0906$ found 335.0906- Anal. Calcd for $\mathrm{C}_{18} \mathrm{H}_{13} \mathrm{~N}_{3} \mathrm{O}_{4}$ : C, 64.47; H, 3.91; N, 12.53. Found: C, 64.12; H, 4.06; N, 12.48.

3-Methyl-2-(methylthio)-7-phenylpyrido[2,3-d]pyrimidin-4(3H)-one 9a. Yellow solid, purified by column chromatography using chloroform as eluant, yield $75 \%$, mp $129-131{ }^{\circ} \mathrm{C} .{ }^{1} \mathrm{H}$ NMR (400 MHz, $\mathrm{CDCl}_{3}$ ): $\delta=2.69$ (s, 3H, $\mathrm{SCH}_{3}$ ), 3.51 (s, 3H, $\mathrm{CH}_{3}$ ), 7.52-7.55 (m, 3H, $\mathrm{H}_{m}, \mathrm{H}_{p}$ ), 8.02 (d, $1 \mathrm{H}, \mathrm{H}_{6}, J=8.28 \mathrm{~Hz}$ ), 8.19 (d, $\left.2 \mathrm{H}, \mathrm{H}_{o}, J=8.28 \mathrm{~Hz}\right), 8.49$ (d, $\left.1 \mathrm{H}, \mathrm{H}_{5}, J=8.20 \mathrm{~Hz}\right) .{ }^{13} \mathrm{C}$ NMR (100 MHz, CDCl $): \delta=15.1\left(\mathrm{SCH}_{3}\right), 30.5\left(\mathrm{NCH}_{3}\right), 112.8\left(\mathrm{C}_{4 \mathrm{a}}\right), 118.5\left(\mathrm{C}_{6}\right), 128.0\left(\mathrm{C}_{o}\right)$, $129.3\left(\mathrm{C}_{m}\right), 130.8\left(\mathrm{C}_{p}\right), 137.7\left(\mathrm{C}_{5}\right), 138.0\left(\mathrm{C}_{i}\right), 156.5\left(\mathrm{C}_{8 \mathrm{a}}\right), 161.2\left(\mathrm{C}_{4}\right), 162.1\left(\mathrm{C}_{2}\right), 162.8\left(\mathrm{C}_{7}\right) . \mathrm{IR}$ $(\mathrm{KBr}) \mathrm{cm}^{-1} 3070$ (CH st), 1682, (C=O st). MS (70 eV) m/z (\%) $283\left(\mathrm{M}^{+}, 66\right), 239$ (25), 238 (100), 237 (27), 209 (31), 208 (32), 196 (17), 140 (29). HR-MS calc. for $\mathrm{C}_{15} \mathrm{H}_{13} \mathrm{~N}_{3} \mathrm{OS} 283.0779$, found 283.0785. Anal. Calcd for $\mathrm{C}_{15} \mathrm{H}_{13} \mathrm{~N}_{3} \mathrm{OS}$ : C, 63.58; H, 4.62; N, 14.83. Found: C, 63.88; $\mathrm{H}$, 4.51; N, 14.78 .

3-Methyl-2-(methylthio)-7-p-tolylpyrido[2,3-d]pyrimidin-4(3H)-one 9b. Yellow solid, purified by column chromatography using chloroform as eluant, yield $70 \%$, mp $189-191{ }^{\circ} \mathrm{C} .{ }^{1} \mathrm{H}$ NMR (400 MHz, DMSO- $\left.d_{6}\right): \delta=2.37\left(\mathrm{~s}, 3 \mathrm{H}, \mathrm{SCH}_{3}\right), 2.50\left(\mathrm{~s}, 3 \mathrm{H}, \mathrm{CH}_{3}\right), 3.25\left(\mathrm{~s}, 3 \mathrm{H}, \mathrm{NCH}_{3}\right)$, 7.35 (d, $2 \mathrm{H}, \mathrm{H}_{m}, J=8.28 \mathrm{~Hz}$ ), 7.81 (d, $1 \mathrm{H}, \mathrm{H}_{6}, J=8.28 \mathrm{~Hz}$ ), 8.05 (d, $2 \mathrm{H}, \mathrm{H}_{o}, J=8.28 \mathrm{~Hz}$ ), 8.31 (d, $\left.1 \mathrm{H}, \mathrm{H}_{5}, J=8.28 \mathrm{~Hz}\right) .{ }^{13} \mathrm{C}$ NMR $\left(100 \mathrm{MHz}, \mathrm{DMSO}-d_{6}\right): \delta=21.4\left(\mathrm{SCH}_{3}\right), 27.6\left(\mathrm{CH}_{3}\right), 40.0$ $\left(\mathrm{NCH}_{3}\right), 108.0\left(\mathrm{C}_{4 \mathrm{a}}\right), 115.5\left(\mathrm{C}_{6}\right), 127.7\left(\mathrm{C}_{o}\right), 130.0\left(\mathrm{C}_{m}\right), 134.6\left(\mathrm{C}_{i}\right), 138.1\left(\mathrm{C}_{5}\right), 140.9\left(\mathrm{C}_{p}\right), 151.1$ $\left(\mathrm{C}_{7}\right), 160.9\left(\mathrm{C}_{4}\right), 162.1\left(\mathrm{C}_{2}\right) . \mathrm{IR}(\mathrm{KBr}) \mathrm{cm}^{-1} 3235$ (CH st), 1716, (C=O st). MS (70 eV) m/z (\%) 297 (M+1,89), 282 (14), 253 (26), 252 (100), 223 (24), 222 (25), 210 (18), 181 (17), 154 (11), 127 (11), 115 (12), 69 (11). HR-MS calc. for $\mathrm{C}_{16} \mathrm{H}_{15} \mathrm{~N}_{3} \mathrm{OS} 297.0936$ found 297.0935. Anal. Calcd for $\mathrm{C}_{16} \mathrm{H}_{15} \mathrm{~N}_{3} \mathrm{OS}$ : C, 64.62; H, 5.08; N, 14.13. Found: C, 64.50; H, 4.99; N, 13.88 .

7-(4-Methoxyphenyl)-3-methyl-2-(methylthio)pyrido[2,3-d]pyrimidin-4(3H)-one 9c. Yellow solid, purified by washing with hot hexanes, yield 70\%, mp $196-198{ }^{\circ} \mathrm{C} .{ }^{1} \mathrm{H} \mathrm{NMR}(400 \mathrm{MHz}$, DMSO- $d_{6}, 373 \mathrm{~K}$ ): $\delta=2.72$ (s, 3H, $\mathrm{SCH}_{3}$ ), 3.53 (s, 3H, $\mathrm{CH}_{3}$ ); 3.86 (s, 3H, $\mathrm{OCH}_{3}$ ), 7.08 (d, 2H, $\mathrm{H}_{o}, J=8.68 \mathrm{~Hz}$ ), 7.89 (d, $1 \mathrm{H}, \mathrm{H}_{6}, J=8.27 \mathrm{~Hz}$ ), 8.11 (d, 2H, $\left.\mathrm{H}_{m}, J=8.69 \mathrm{~Hz}\right), 8.43$ (d, 1H, $\mathrm{H}_{5}, J$ $=8.27 \mathrm{~Hz}) \cdot{ }^{13} \mathrm{C}$ NMR $\left(100 \mathrm{MHz}, \mathrm{DMSO}-d_{6}, 373 \mathrm{~K}\right): \delta=14.1\left(\mathrm{SCH}_{3}\right), 29.6\left(\mathrm{NCH}_{3}\right), 54.9$ $\left(\mathrm{OCH}_{3}\right), 111.2\left(\mathrm{C}_{4 \mathrm{a}}\right), 113.9\left(\mathrm{C}_{o}\right), 116.8\left(\mathrm{C}_{6}\right), 128.5\left(\mathrm{C}_{m}\right), 129.8\left(\mathrm{C}_{i}\right), 136.3\left(\mathrm{C}_{5}\right), 155.7\left(\mathrm{C}_{8 \mathrm{a}}\right)$, $160.4\left(\mathrm{C}_{4}\right), 160.9\left(\mathrm{C}_{7}\right), 161.0\left(\mathrm{C}_{2}\right), 161.6\left(\mathrm{C}_{p}\right) . \mathrm{IR}(\mathrm{KBr}) \mathrm{cm}^{-1}$ 1677, (C=O st). MS (70 eV) m/z (\%) 313 (M+100), 298 (15), 269 (26), 268 (87), 267 (54), 239 (214), 238 (27), 226 (20), 197 (19). HR-MS calc. for $\mathrm{C}_{16} \mathrm{H}_{15} \mathrm{~N}_{3} \mathrm{O}_{2} \mathrm{~S} 313.0885$ found 313.0884. Anal. Calcd for $\mathrm{C}_{16} \mathrm{H}_{15} \mathrm{~N}_{3} \mathrm{O}_{2} \mathrm{~S}$ : C, 61.32; H, 4.82; N, 13.41. Found: C, 61.50; H, 4.59; N, 13.58.

7-(3,4,5-Trimethoxyphenyl)-3-methyl-2-(methylthio)pyrido[2,3-d]pyrimidin-4(3H)-one 9d. Yellow solid, purified by washing with hot hexanes, yield 70\%, mp 211-213 ${ }^{\circ} \mathrm{C} .{ }^{1} \mathrm{H}$ NMR (400 MHz, DMSO- $\left.d_{6}\right): \delta=2.72\left(\mathrm{~s}, 3 \mathrm{H}, \mathrm{SCH}_{3}\right), 3.52$ (s, 3H, $\left.\mathrm{CH}_{3}\right) ; 3.76\left(\mathrm{~s}, 3 \mathrm{H}, \mathrm{OCH}_{3}\right), 3.93$ (s, 6H, $\left.\mathrm{OCH}_{3}\right), 7.48\left(\mathrm{~s}, 2 \mathrm{H}, \mathrm{H}_{o}\right), 8.08\left(\mathrm{~d}, 1 \mathrm{H}, \mathrm{H}_{6}, J=8.48 \mathrm{~Hz}\right), 8.47\left(\mathrm{~d}, 1 \mathrm{H}, \mathrm{H}_{5}, J=8.27 \mathrm{~Hz}\right) .{ }^{13} \mathrm{C} \mathrm{NMR}$ (100 MHz, DMSO- $\left.d_{6}\right): \delta=14.8\left(\mathrm{SCH}_{3}\right), 30.1\left(\mathrm{NCH}_{3}\right), 56.1\left(\mathrm{OCH}_{3}\right), 60.2\left(\mathrm{OCH}_{3}\right), 105.0\left(\mathrm{C}_{o}\right)$, $112.2\left(\mathrm{C}_{4 \mathrm{a}}\right), 118.4\left(\mathrm{C}_{6}\right), 132.8\left(\mathrm{C}_{i}\right), 137.2\left(\mathrm{C}_{5}\right), 139.8\left(\mathrm{C}_{p}\right), 153.2\left(\mathrm{C}_{m}\right), 155.9\left(\mathrm{C}_{8 \mathrm{a}}\right), 160.8\left(\mathrm{C}_{7}\right)$, $161.1\left(\mathrm{C}_{4}\right), 162.5\left(\mathrm{C}_{2}\right)$. IR (KBr) cm ${ }^{-1}$ 1679, (C=O st). MS (70 eV) m/z (\%)373 ( $\left.\mathrm{M}^{+}, 100\right), 372$ (30), 358 (37), 327 (48), 312 (30), 299 (34), 142 (20), 128 (26), 88 (43), 44 (31). HR-MS calc. 
for $\mathrm{C}_{18} \mathrm{H}_{19} \mathrm{~N}_{3} \mathrm{O}_{4} \mathrm{~S} 373.1096$ found 373.1084. Anal. Calcd for $\mathrm{C}_{18} \mathrm{H}_{19} \mathrm{~N}_{3} \mathrm{O}_{4} \mathrm{~S}$ : C, 57.89; H, 5.13; N, 11.25. Found: C, 57.54; H, 4.96; N, 11.49.

7-(1,1'-Biphenyl-4yl)-3-methyl-2-(methylthio)pyrido[2,3-d]pyrimidin-4(3H)-one 9e. Yellow solid, purified by column chromatography using chloroform as eluant, yield 50\%, mp 222-224 ${ }^{\circ} \mathrm{C} .{ }^{1} \mathrm{H}$ NMR (400 MHz, $\mathrm{CDCl}_{3}$ ): $\delta=2.81$ (s, 3H, $\mathrm{SCH}_{3}$ ), 3.63 (s, 3H, $\left.\mathrm{NCH}_{3}\right), 7.38\left(\mathrm{t}, 1 \mathrm{H}, \mathrm{H}_{p}, J\right.$ $=7.28 \mathrm{~Hz}$ ), 7.47 (t, $\left.2 \mathrm{H}, \mathrm{H}_{m}, J=7.53 \mathrm{~Hz}\right), 7.66$ (d, 2H, $\left.\mathrm{H}_{o}, J=7.28 \mathrm{~Hz}\right), 7.83\left(\mathrm{~d}, 1 \mathrm{H}, \mathrm{H}_{6}, J=8.28\right.$ $\mathrm{Hz}$ ), 7.74 (d, $2 \mathrm{H}, \mathrm{H}_{m}, J=8.28 \mathrm{~Hz}$ ), 8.24 (d, 2H, $\left.\mathrm{H}_{o}, J=8.28 \mathrm{~Hz}\right), 8.60$ (d, 1H, $\left.\mathrm{H}_{5}, J=8.03 \mathrm{~Hz}\right)$. ${ }^{13} \mathrm{C}$ NMR (100 MHz, $\left.\mathrm{CDCl}_{3}\right): \delta=15.0\left(\mathrm{SCH}_{3}\right), 30.1\left(\mathrm{NCH}_{3}\right), 112.3\left(\mathrm{C}_{4 \mathrm{a}}\right), 118.0\left(\mathrm{C}_{6}\right), 126.8\left(\mathrm{C}_{o}\right)$, $127.2\left(\mathrm{C}_{o}\right), 127.5\left(\mathrm{C}_{p}\right), 128.1\left(\mathrm{C}_{m}\right), 128.6\left(\mathrm{C}_{m}\right), 136.7\left(\mathrm{C}_{i}\right), 137.2\left(\mathrm{C}_{5}\right), 140.0\left(\mathrm{C}_{i}\right), 142.7\left(\mathrm{C}_{p}\right)$, $156.6\left(\mathrm{C}_{8 \mathrm{a}}\right), 161.6\left(\mathrm{C}_{2}\right), 162.7(\mathrm{C} 7), 185.3\left(\mathrm{C}_{4}\right) . \mathrm{IR}(\mathrm{KBr}) \mathrm{cm}^{-1} 2922(\mathrm{CH}, s t), 1676(\mathrm{C}=\mathrm{O} s t)$. MS (70 eV) $m / z(\%)=359\left(\mathrm{M}^{+}, 100\right), 314$ (91), 284 (24), 243 (21). HR-MS calc. for $\mathrm{C}_{21} \mathrm{H}_{17} \mathrm{~N}_{3} \mathrm{OS}$ 359.1092, found 359.1094. Anal. Calcd for $\mathrm{C}_{21} \mathrm{H}_{17} \mathrm{~N}_{3} \mathrm{OS}$ : C, 70.17; H, 4.77; N, 11.69. Found: C, 68.99; H, 4.56; N, 11.29.

7-(4-Hydroxyphenyl)-3-methyl-2-(methylthio)pyrido[2,3-d]pyrimidin-4(3H)-one 9f. Yellow solid, yield 80\%, mp 249-251 ${ }^{\circ} \mathrm{C} .{ }^{1} \mathrm{H}$ NMR (400 $\mathrm{MHz} \mathrm{CDCl}_{3}$ ): $\delta=2.68$ (s, 3H, $\mathrm{SCH}_{3}$ ), 3.49 (s, $3 \mathrm{H}, \mathrm{CH}_{3}$ ); 6.91 (d, 2H, $\mathrm{H}_{m}, J=8.53 \mathrm{~Hz}$ ), 7.91 (d, $1 \mathrm{H}, \mathrm{H}_{6}, J=8.28 \mathrm{~Hz}$ ), 8.06 (d, 2H, $\mathrm{H}_{o}, J=8.53$ $\mathrm{Hz}), 8.43\left(\mathrm{~d}, 1 \mathrm{H}, \mathrm{H}_{5}, J=8.29 \mathrm{~Hz}\right) .{ }^{13} \mathrm{C} \mathrm{NMR}\left(100 \mathrm{MHz}, \mathrm{CDCl}_{3}\right): \delta=15.3\left(\mathrm{SCH}_{3}\right), 30.6\left(\mathrm{NCH}_{3}\right)$, $111.8\left(\mathrm{C}_{4 \mathrm{a}}\right), 116.2\left(\mathrm{C}_{m}\right), 117.8\left(\mathrm{C}_{6}\right), 129.9\left(\mathrm{C}_{o}\right), 137.9\left(\mathrm{C}_{5}\right), 156.3\left(\mathrm{C}_{8 \mathrm{a}}\right), 160.5\left(\mathrm{C}_{4}\right), 161.1\left(\mathrm{C}_{7}\right)$, $161.6\left(\mathrm{C}_{2}\right), 162.9\left(\mathrm{C}_{p}\right)$. IR (KBr) cm $3228(\mathrm{OH}, s t), 1598$, (C=O st). MS (70 eV) m/z (\%) 299 (M+100), 255 (28), 254 (97), 253 (49), 225 (32), 224 (35), 212 (27), 183 (26). HR-MS calc. for $\mathrm{C}_{15} \mathrm{H}_{13} \mathrm{~N}_{3} \mathrm{O}_{2} \mathrm{~S}$ 299.0728, found 299.0734. Anal. Calcd for $\mathrm{C}_{15} \mathrm{H}_{13} \mathrm{~N}_{3} \mathrm{O}_{2} \mathrm{~S}: \mathrm{C}, 60.18 ; \mathrm{H}, 4.38 ; \mathrm{N}$, 14.04. Found: C, 60.58; H, 4.48; N, 14.34 .

3-Methyl-2-(methylthio)-7-(4-nitrophenyl)pyrido[2,3-d]pyrimidin-4(3H)-one 9g. Yellow solid, recrystallized from DMF, yield 70\%, mp 272-274 ${ }^{\circ} \mathrm{C} .{ }^{1} \mathrm{H}$ NMR (400 MHz, DMSO- $\left.d_{6}\right): \delta=$ 2.74 (s, 3H, $\mathrm{SCH}_{3}$ ), 3.56 (s, 3H, $\mathrm{CH}_{3}$ ), 8.10 (d, $1 \mathrm{H}, \mathrm{H}_{6}, J=8.03 \mathrm{~Hz}$ ), 8.35 (d, 2H, $\mathrm{H}_{o}, J=8.79$ $\mathrm{Hz}), 8.45$ (d, $\left.2 \mathrm{H}, \mathrm{H}_{m}, J=8.79 \mathrm{~Hz}\right), 8.58$ (d, $\left.1 \mathrm{H}, \mathrm{H}_{5}, J=8.03 \mathrm{~Hz}\right) .{ }^{13} \mathrm{C}$ NMR (100 MHz, DMSO$\left.d_{6}\right): \delta=15.3\left(\mathrm{SCH}_{3}\right), 30.6\left(\mathrm{NCH}_{3}\right), 113.7\left(\mathrm{C}_{4 \mathrm{a}}\right), 119.3\left(\mathrm{C}_{6}\right), 124.4\left(\mathrm{C}_{m}\right), 129.1\left(\mathrm{C}_{o}\right), 138.2\left(\mathrm{C}_{5}\right)$, $143.7\left(\mathrm{C}_{i}\right), 148.8\left(\mathrm{C}_{p}\right), 156.5\left(\mathrm{C}_{8 \mathrm{a}}\right), 159.5\left(\mathrm{C}_{4}\right), 161.2\left(\mathrm{C}_{2}\right), 163.4\left(\mathrm{C}_{7}\right) . \mathrm{IR}(\mathrm{KBr}) \mathrm{cm}^{-1} 3120(\mathrm{CH}$ $s t)$, 1675, (C=O st). MS (70 eV) m/z (\%)328 (M+ 54), 313 (12), 284 (26), 283 (100), 254 (21), 253 (18), 166 (11), 97 (15), 69 (20), 43 (17). HR-MS calc. for $\mathrm{C}_{15} \mathrm{H}_{12} \mathrm{~N}_{4} \mathrm{O}_{3} \mathrm{~S} 328.0630$ found 328.0617. Anal. Calcd for $\mathrm{C}_{15} \mathrm{H}_{12} \mathrm{~N}_{4} \mathrm{O}_{3} \mathrm{~S}$ : C, 54.87; H, 3.68; N, 17.06. Found: C, 54.32; H, 3.76; $\mathrm{N}, 17.22$.

7-(4-Chlorophenyl)-3-methyl-2-(methylthio)pyrido[2,3-d]pyrimidin-4(3H)-one 9h. Yellow solid, was recrystallized from a mixture of ethanol - DMF, yield 70\%, mp $219.0-221.0{ }^{\circ} \mathrm{C} .{ }^{1} \mathrm{H}$ NMR (400 MHz, $\mathrm{CDCl}_{3}$ ): $\delta=2.70$ (s, 3H, $\mathrm{SCH}_{3}$ ), 3.52 (s, 3H, $\mathrm{CH}_{3}$ ); 7.53 (d, 2H, $\mathrm{CH}_{o}, J=8.53$ $\mathrm{Hz}$ ), 7.91 (d, $\left.1 \mathrm{H}, \mathrm{H}_{6}, J=8.29 \mathrm{~Hz}\right), 8.15$ (d, $\left.2 \mathrm{H}, \mathrm{CH}_{m}, J=8.53 \mathrm{~Hz}\right), 8.47$ (d, $\left.1 \mathrm{H}, \mathrm{H}_{5}, J=8.29 \mathrm{~Hz}\right)$.

${ }^{13} \mathrm{C}$ NMR (100 MHz, $\left.\mathrm{CDCl}_{3}\right): \delta=14.5\left(\mathrm{SCH}_{3}\right), 30.0\left(\mathrm{CH}_{3}\right), 112.3\left(\mathrm{C}_{4 \mathrm{a}}\right), 117.7\left(\mathrm{C}_{6}\right), 128.6\left(\mathrm{C}_{o}\right)$, $129.0\left(\mathrm{C}_{m}\right), 135.2\left(\mathrm{C}_{i}\right), 136.4\left(\mathrm{C}_{p}\right), 137.1\left(\mathrm{C}_{5}\right), 156.0\left(\mathrm{C}_{8 \mathrm{a}}\right), 160.4\left(\mathrm{C}_{4}\right), 160.8\left(\mathrm{C}_{7}\right), 162.3\left(\mathrm{C}_{2}\right) . \mathrm{IR}$ $(\mathrm{KBr}) \mathrm{cm}^{-1}$ 1687, (C=O st). MS (70 eV) m/z (\%) 319/317 ( $\left.\mathrm{M}^{+2} / \mathrm{M}^{+}, 22 / 58\right), 274 / 272$ (35/100), 271 (26), 243 (24), 232/230 (5/15), 166/165 (19/5). HR-MS calc. for $\mathrm{C}_{15} \mathrm{H}_{12} \mathrm{ClN}_{3} \mathrm{OS} 317.0390$ 
found 317.0399. Calcd for $\mathrm{C}_{15} \mathrm{H}_{12} \mathrm{ClN}_{3} \mathrm{OS}$ : C, 56.69; H, 3.81; N, 13.22. Found: C, 56.22; $\mathrm{H}$, 3.86; N, 13.12.

7-(4-Bromophenyl)-3-methyl-2-(methylthio)pyrido[2,3-d]pyrimidin-4(3H)-one 9i. Yellow solid, purified by washing with hot hexanes, yield $80 \%$, mp $206-208{ }^{\circ} \mathrm{C}$. ${ }^{1} \mathrm{H}$ NMR $(400 \mathrm{MHz}$, DMSO- $d_{6}$ ): $\delta=2.68$ (s, $3 \mathrm{H}, \mathrm{SCH}_{3}$ ), 3.50 (s, 3H, $\mathrm{CH}_{3}$ ); 7.73 (d, 2H, $\mathrm{H}_{o}, J=8.29 \mathrm{~Hz}$ ), 8.02 (d, $1 \mathrm{H}$, $\left.\mathrm{H}_{6}, J=8.28 \mathrm{~Hz}\right), 8.15$ (d, $\left.2 \mathrm{H}, \mathrm{H}_{m}, J=8.54 \mathrm{~Hz}\right), 8.48$ (d, $\left.1 \mathrm{H}, \mathrm{H}_{5}, J=8.29 \mathrm{~Hz}\right) .{ }^{13} \mathrm{C}$ NMR $(100$ MHz, DMSO- $\left.d_{6}\right): \delta=15.3\left(\mathrm{SCH}_{3}\right), 30.7\left(\mathrm{CH}_{3}\right), 113.1\left(\mathrm{C}_{4 \mathrm{a}}\right), 118.4\left(\mathrm{C}_{6}\right), 124.7\left(\mathrm{C}_{p}\right), 129.9\left(\mathrm{C}_{o}\right)$, $132.4\left(\mathrm{C}_{m}\right), 137.1\left(\mathrm{C}_{i}\right), 137.9\left(\mathrm{C}_{5}\right), 156.6\left(\mathrm{C}_{8 \mathrm{a}}\right), 160.7\left(\mathrm{C}_{2}\right), 161.3\left(\mathrm{C}_{7}\right), 163.0\left(\mathrm{C}_{4}\right) . \mathrm{IR}(\mathrm{KBr}) \mathrm{cm}^{-1}$ 1689, (C=O st). MS (70 eV) m/z (\%)363/361 ( $\left.\mathrm{M}^{+2} / \mathrm{M}^{+}, 54 / 54\right), 317 / 315(93 / 100), 288 / 286$ (19/20), 246/244 (19/15). HR-MS calc. for $\mathrm{C}_{15} \mathrm{H}_{12} \mathrm{BrN}_{3} \mathrm{OS} 360.9884$, found 360.8978. Anal. Calcd for $\mathrm{C}_{15} \mathrm{H}_{12} \mathrm{BrN}_{3} \mathrm{OS}$ : C, 49.73; H, 3.34; N, 11.60. Found: C, 51.12; H, 3.45; N, 11.44 .

7-(4-Fluorophenyl)-3-methyl-2-(methylthio)pyrido[2,3-d]pyrimidin-4(3H)-one 9j. Yellow solid, purified by column chromatography using chloroform as eluant, yield 70\%, mp 202-204 ${ }^{\circ} \mathrm{C}$. ${ }^{1} \mathrm{H}$ NMR (400 MHz, DMSO- $d_{6}$ ): $\delta=2.69$ (s, 3H, $\mathrm{SCH}_{3}$ ), 3.51 (s, 3H, $\mathrm{CH}_{3}$ ); 7.36 (t, 2H, $\mathrm{H}_{m}$, $J=8.78 \mathrm{~Hz}$ ), 8.02 (d, $\left.1 \mathrm{H}, \mathrm{H}_{6}, J=8.28 \mathrm{~Hz}\right), 8.25-8.29$ (m, 2H, $\left.\mathrm{H}_{o}\right), 8.48$ (d, $\left.1 \mathrm{H}, \mathrm{H}_{5}, J=8.29 \mathrm{~Hz}\right)$. ${ }^{13} \mathrm{C}$ NMR (100 MHz, DMSO- $\left.d_{6}\right): \delta=15.2\left(\mathrm{SCH}_{3}\right), 30.6\left(\mathrm{CH}_{3}\right), 112.7\left(\mathrm{C}_{4 \mathrm{a}}\right), 116.4\left(\mathrm{C}_{o}\right), 118.3$ $\left(\mathrm{C}_{6}\right), 130.2\left(\mathrm{C}_{m}\right), 134.5\left(\mathrm{C}_{p}\right), 137.8\left(\mathrm{C}_{5}\right), 156.6\left(\mathrm{C}_{8 \mathrm{a}}\right), 160.9\left(\mathrm{C}_{4}\right), 161.3\left(\mathrm{C}_{2}\right), 162.8\left(\mathrm{C}_{7}\right) . \mathrm{IR}(\mathrm{KBr})$ $\mathrm{cm}^{-1} 1664$ (C=O st). MS (70 eV) m/z (\%) 301 ( $\left.\mathrm{M}^{+}, 74\right), 271$ (16), 257 (27), 256 (100), 255 (31), 227 (29), 226 (31), 214 (23), 185 (20), 158 (29). HR-MS calc. for $\mathrm{C}_{15} \mathrm{H}_{12} \mathrm{FN}_{3} \mathrm{OS}$ 301.0685, found 301.0688. Anal. Calcd for $\mathrm{C}_{15} \mathrm{H}_{12} \mathrm{FN}_{3} \mathrm{OS}$ : C, 59.79; H, 4.01; N, 13.94. Found: C, 59.12; H, 4.12; $\mathrm{N}, 13.06$.

3-Methyl-2-(methylthio)-7-(2-oxo-2H-chromen-3-yl)pyrido[2,3-d]pyrimidin-4(3H)-one 9k. Yellow solid, yield 70\%, recrystallized from ethanol, mp 217-219 ${ }^{\circ} \mathrm{C}$. ${ }^{1} \mathrm{H}$ NMR (400 MHz, $\left.\mathrm{CDCl}_{3}\right): \delta=2.79\left(\mathrm{~s}, 3 \mathrm{H}, \mathrm{SCH}_{3}\right), 3.64\left(\mathrm{~s}, 3 \mathrm{H}, \mathrm{NCH}_{3}\right), 7.34\left(\mathrm{t}, 1 \mathrm{H}, \mathrm{H}_{\text {aryl }}\right), 7.38\left(\mathrm{~d}, 1 \mathrm{H}, \mathrm{H}_{\text {aryl }}, J=\right.$ $8.29 \mathrm{~Hz}$ ), 7.59 (t, 1H, $\mathrm{H}_{\text {aryl }}$ ), 7.68 (d, 2H, $\mathrm{H}_{\text {aryl }}, J=7.65 \mathrm{~Hz}$ ), 8.47 (d, $\left.\mathrm{H}_{6}, J=8.28 \mathrm{~Hz}\right), 8.61$ (d, $\mathrm{H}_{5}, J=8.28 \mathrm{~Hz}$ ), 9.00 (s, 1H, CH). $\left.{ }^{13} \mathrm{C} \mathrm{NMR} \mathrm{(100} \mathrm{MHz,} \mathrm{CDCl}_{3}\right): \delta=15.3\left(\mathrm{SCH}_{3}\right), 30.5$ $\left(\mathrm{NCH}_{3}\right), 113.7\left(\mathrm{C}_{4 \mathrm{a}}\right), 116.5\left(\mathrm{C}_{6}\right), 119.2\left(\mathrm{C}_{q}\right), 121.6\left(\mathrm{C}_{\text {aryl }}\right), 121.6\left(\mathrm{C}_{\text {aryl }}\right), 124.2\left(\mathrm{C}_{\text {q-aryl }}\right), 124.8$ $\left(\mathrm{C}_{\text {aryl }}\right), 129.3\left(\mathrm{C}_{\text {aryl }}\right), 133.0\left(\mathrm{C}_{\text {aryl }}\right), 137.8\left(\mathrm{C}_{5}\right), 145.4(\mathrm{CH}), 154.3\left(\mathrm{C}_{7}\right), 156.3\left(\mathrm{C}_{2}\right), 157.4\left(\mathrm{C}_{4}\right)$, $160.8\left(\mathrm{C}_{\text {q-aryl }}\right), 161.5(\mathrm{C}=\mathrm{O}), 163.1\left(\mathrm{C}_{8 \mathrm{a}}\right) . \mathrm{IR}(\mathrm{KBr}) \mathrm{cm}^{-1} 1729,1681,(\mathrm{C}=\mathrm{O} s t) . \mathrm{MS}(70 \mathrm{eV}) \mathrm{m} / z$ (\%) 353 ( $\left.\mathrm{M}^{+}, 5\right), 352$ (16), 351 (79), 336 (14), 307 (25), 306 (100), 277 (32), 275 (22), 264 (14). HR-MS calc. for $\mathrm{C}_{18} \mathrm{H}_{13} \mathrm{~N}_{3} \mathrm{O}_{3} \mathrm{~S}$ 351.0678, found 351.0683. Anal. Calcd for $\mathrm{C}_{18} \mathrm{H}_{13} \mathrm{~N}_{3} \mathrm{O}_{3} \mathrm{~S}$ : C, 61.53; H, 3.73; N, 11.96. Found: C, 59.96; H, 3.92; N, 13.14.

3-Methyl-7-phenylpyrido[2,3-d]pyrimidine-2,4(1H,3H)-dione 10a. White solid, yield 70\%, m.p 291-293 ${ }^{\circ} \mathrm{C}$. ${ }^{1} \mathrm{H}$ NMR (400 MHz, DMSO- $d_{6}$ ): $\delta=3.25$ (s, $3 \mathrm{H}, \mathrm{NCH}_{3}$ ), 7.51-7.55 (m, 3H, $\mathrm{H}_{m}$, $\mathrm{H}_{p}$ ), $7.82\left(\mathrm{~d}, 1 \mathrm{H}, \mathrm{H}_{6}, J=8.03 \mathrm{~Hz}\right), 8.13\left(\mathrm{~d}, 2 \mathrm{H}, \mathrm{H}_{o}, J=7.79 \mathrm{~Hz}\right), 8.33$ (d, 1H, $\left.\mathrm{H}_{5}, J=8.28 \mathrm{~Hz}\right)$, 11.96 (s, 1H, NH). ${ }^{13} \mathrm{C}$ NMR (100 MHz, DMSO- $\left.d_{6}\right): \delta=27.5\left(\mathrm{NCH}_{3}\right), 108.4\left(\mathrm{C}_{4 \mathrm{a}}\right), 115.8\left(\mathrm{C}_{6}\right)$, $127.7\left(\mathrm{C}_{o}\right), 129.3\left(\mathrm{C}_{m}\right), 130.9\left(\mathrm{C}_{p}\right), 137.4\left(\mathrm{C}_{i}\right), 138.1\left(\mathrm{C}_{5}\right), 151.1(\mathrm{C}-7), 151.2\left(\mathrm{C}_{8 \mathrm{a}}\right), 160.9\left(\mathrm{C}_{4}\right)$, $162.0\left(\mathrm{C}_{2}\right)$. IR $(\mathrm{KBr}) \mathrm{cm}^{-1} 3150(\mathrm{NH}, s t), 1726(\mathrm{C}=\mathrm{O} s t)$. The mass spectrum shows the following peaks: MS (70 eV) m/z (\%) = 253 (M+1 100), 224 (24), 196 (33), 169 (26), 140 (19). HRMS calc. 
for $\mathrm{C}_{14} \mathrm{H}_{11} \mathrm{~N}_{3} \mathrm{O}_{2}$ 253.0851, found 253.0856. Anal. Calcd for $\mathrm{C}_{14} \mathrm{H}_{11} \mathrm{~N}_{3} \mathrm{O}_{2}$ : C, 66.40; $\mathrm{H}, 4.38 ; \mathrm{N}$, 16.59. Found: C, 66.78; H, 4.56; N, 16.52 .

3-Methyl-7-p-tolylpyrido[2,3-d]pyrimidine-2,4(1H,3H)-dione 10b. White solid, yield 60\%, m.p. $>300{ }^{\circ} \mathrm{C} .{ }^{1} \mathrm{H}$ NMR (400 MHz, DMSO- $\left.d_{6}, 393 \mathrm{~K}\right): \delta=2.38$ (s, 3H, $\mathrm{CH}_{3}$ ), 3.29 (s, 3H, $\mathrm{NCH}_{3}$ ), 7.31 (d, 2H, Ho, $J=8.00 \mathrm{~Hz}$ ), 7.70 (d, $1 \mathrm{H}, \mathrm{H}_{6}, J=8.28 \mathrm{~Hz}$ ), 8.00 (d, 2H, $\mathrm{H}_{m}, J=8.00$ $\mathrm{Hz}$ ), 8.29 (d, $\left.1 \mathrm{H}, \mathrm{H}_{5}, J=8.28 \mathrm{~Hz}\right), 11.32$ (s, $\left.1 \mathrm{H}, \mathrm{NH}\right) .{ }^{13} \mathrm{C}$ NMR (100 MHz, DMSO- $\left.d_{6}, 393 \mathrm{~K}\right): \delta$ $=20.1\left(\mathrm{CH}_{3}\right), 26.2\left(\mathrm{NCH}_{3}\right), 107.0\left(\mathrm{C}_{4 \mathrm{a}}\right), 114.4\left(\mathrm{C}_{6}\right), 126.6\left(\mathrm{C}_{m}\right), 128.7\left(\mathrm{C}_{o}\right), 134.1\left(\mathrm{C}_{i}\right), 136.8$ $\left(\mathrm{C}_{5}\right), 139.6\left(\mathrm{C}_{p}\right), 150.0(\mathrm{C}-7), 160.4\left(\mathrm{C}_{4}\right), 161.1\left(\mathrm{C}_{2}\right), 150.1\left(\mathrm{C}_{8 \mathrm{a}}\right) . \mathrm{IR}(\mathrm{KBr}) \mathrm{cm}^{-1} 3122(\mathrm{NH}, s t)$, 1712 (C=O st). MS (70 eV) m/z (\%) 267 (M+100), 238 (16), 210 (26), 183 (17). HRMS calc. For $\mathrm{C}_{15} \mathrm{H}_{13} \mathrm{~N}_{3} \mathrm{O}_{2} 267.1008$ found 267.1009. Anal. Calcd for $\mathrm{C}_{15} \mathrm{H}_{13} \mathrm{~N}_{3} \mathrm{O}_{2}$ : C, 67.40; $\mathrm{H}, 4.90 ; \mathrm{N}$, 15.72. Found: C, 66.96; H, 4.52; N, 15.76.

7-(4-Methoxyphenyl)-3-methylpyrido[2,3-d]pyrimidine-2,4(1H,3H)-dione 10c. Yellow solid, recrystallized from DMF, yield 70\%, mp $>300{ }^{\circ} \mathrm{C}$. ${ }^{1} \mathrm{H}$ NMR (400 MHz, DMSO- $\left.d_{6}\right): \delta=3.24$ (s, $3 \mathrm{H}, \mathrm{NCH}_{3}$ ), 3.83 (s, 3H, $\mathrm{OCH}_{3}$ ), 7.57 (d, 2H, $\left.\mathrm{H}_{m}, J=8.78 \mathrm{~Hz}\right), 7.75$ (d, 1H, $\mathrm{H}_{6}, J=8.28 \mathrm{~Hz}$ ), 8.12 (d, $\left.2 \mathrm{H}, \mathrm{H}_{o}, J=7.53 \mathrm{~Hz}\right), 8.26$ (d, $\left.1 \mathrm{H}, \mathrm{H}_{5}, J=8.28 \mathrm{~Hz}\right), 11.68$ (s, 1H, NH). ${ }^{13} \mathrm{C}$ NMR (100 MHz, DMSO-d $\left.d_{6}\right): \delta=26.5\left(\mathrm{NCH}_{3}\right), 55.8\left(\mathrm{OCH}_{3}\right), 107.1\left(\mathrm{C}_{4 \mathrm{a}}\right), 114.8\left(\mathrm{C}_{o}\right), 115.0\left(\mathrm{C}_{6}\right), 129.4$ $\left(\mathrm{C}_{m}\right), 129.5\left(\mathrm{C}_{i}\right), 137.9\left(\mathrm{C}_{5}\right), 149.8\left(\mathrm{C}_{7}\right), 150.7\left(\mathrm{C}_{8 \mathrm{a}}\right), 155.8\left(\mathrm{C}_{p}\right), 160.2\left(\mathrm{C}_{4}\right), 161.3\left(\mathrm{C}_{2}\right), . \mathrm{IR}$ (KBr) $\mathrm{cm}^{-1}$ 3280, (NH st), 1720, (C=O st). MS (70 eV) m/z (\%) $283\left(\mathrm{M}^{+}, 100\right), 254$ (10), 226 (20), 155 (8). HR-MS calc. for $\mathrm{C}_{15} \mathrm{H}_{13} \mathrm{~N}_{3} \mathrm{O}_{3}$ 283.0957, found 283.0966. Anal. Calcd for $\mathrm{C}_{15} \mathrm{H}_{13} \mathrm{~N}_{3} \mathrm{O}_{3}$ : C, 63.60; H, 4.63; N, 14.83. Found: C, 64.06; H, 4.53; N, 14.95.

7-(3,4,5-Trimethoxyphenyl)-3-methylpyrido[2,3-d]pyrimidine-2,4(1H,3H)-dione 10d. Brown solid, recrystallized from DMF, yield 60\%, mp 297-299 ${ }^{\circ} \mathrm{C} .{ }^{1} \mathrm{H}$ NMR (400 MHz, DMSO- $\left.d_{6}\right): \delta=$ 3.24 (s, 3H, $\left.\mathrm{NCH}_{3}\right), 3.73$ (s, 3H, $\left.\mathrm{OCH}_{3}\right), 3.88\left(\mathrm{~s}, 6 \mathrm{H}, \mathrm{OCH}_{3}\right), 7.46$ (s, 2H, $\left.\mathrm{H}_{o}\right), 7.86\left(\mathrm{~d}, 1 \mathrm{H}, \mathrm{H}_{6}, J\right.$ $=8.28 \mathrm{~Hz}), 8.28\left(\mathrm{~d}, 1 \mathrm{H}, \mathrm{H}_{5}, J=8.28 \mathrm{~Hz}\right), 11.90(\mathrm{~s}, 1 \mathrm{H}, \mathrm{NH}) .{ }^{13} \mathrm{C}$ NMR $\left(100 \mathrm{MHz}, \mathrm{DMSO}-d_{6}\right): \delta$ $=27.6\left(\mathrm{NCH}_{3}\right), 56.5\left(\mathrm{C}_{m}-\mathrm{OCH}_{3}\right), 60.6\left(\mathrm{C}_{p}-\mathrm{OCH}_{3}\right), 105.3\left(\mathrm{C}_{o}\right), 108.1\left(\mathrm{C}_{4 \mathrm{a}}\right), 115.8\left(\mathrm{C}_{6}\right), 132.8$ $\left(\mathrm{C}_{i}\right), 137.9\left(\mathrm{C}_{5}\right), 140.3\left(\mathrm{C}_{p}\right), 151.0\left(\mathrm{C}_{7}\right), 151.1\left(\mathrm{C}_{8 \mathrm{a}}\right), 153.6\left(\mathrm{C}_{m}\right), 160.2\left(\mathrm{C}_{4}\right), 162.1\left(\mathrm{C}_{2}\right) . \mathrm{IR}(\mathrm{KBr})$ $\mathrm{cm}^{-1}$ 3053, (NH st), 1715, (C=O st). MS (70 eV) m/z (\%) $343\left(\mathrm{M}^{+}, 100\right), 328$ (46), 270 (22), 213 (17), 257 (17). HR-MS calc. for $\mathrm{C}_{17} \mathrm{H}_{17} \mathrm{~N}_{3} \mathrm{O}_{5}$ 343.1168, found 343.1169. Anal. Calcd for $\mathrm{C}_{17} \mathrm{H}_{17} \mathrm{~N}_{3} \mathrm{O}_{5}$ : C, 59.47; H, 4.99; N: 12.24. Found: C, 58.19; H, 5.03; N, 13.07.

7-(1,1'-Biphenyl-4-yl)-3-methylpyrido[2,3-d]pyrimidine-2,4(1H,3H)-dione 10e. Yellow solid, yield $40 \%$, mp $>300{ }^{\circ} \mathrm{C} .{ }^{1} \mathrm{H}$ NMR (400 MHz, DMSO- $\left.d_{6}, 393 \mathrm{~K}\right): \delta=3.30\left(\mathrm{~s}, 3 \mathrm{H}, \mathrm{NCH}_{3}\right), 7.38(\mathrm{t}$, $\left.1 \mathrm{H}, \mathrm{H}_{p}\right), 7.71-7.52\left(\mathrm{~m}, 7 \mathrm{H}, \mathrm{H}_{\text {arom }}\right), 8.22\left(\mathrm{~d}, 2 \mathrm{H}, \mathrm{H}_{\text {arom }}, J=8.69 \mathrm{~Hz}\right), 8.35$ (d, $1 \mathrm{H}, \mathrm{H}_{5}, J=8.0 .7$ $\mathrm{Hz}), 11.38(s, 1 \mathrm{H}, \mathrm{NH}) .{ }^{13} \mathrm{C}$ NMR $\left(100 \mathrm{MHz}, \mathrm{DMSO}-d_{6}, 393 \mathrm{~K}\right): \delta=26.3\left(\mathrm{NCH}_{3}\right), 114.7\left(\mathrm{C}_{6}\right)$, $126.0\left(\mathrm{C}_{o}\right), 126.4\left(\mathrm{C}_{o}\right), 127.1\left(\mathrm{C}_{p}\right), 127.2\left(\mathrm{C}_{m}\right), 128.2\left(\mathrm{C}_{m}\right), 136.9\left(\mathrm{C}_{5}\right), 141.7\left(\mathrm{C}_{\mathrm{q}}\right), 142.2\left(\mathrm{C}_{\mathrm{q}}\right)$, $149.9\left(\mathrm{C}_{7}\right), 150.2\left(\mathrm{C}_{8 \mathrm{a}}\right), 152.1\left(\mathrm{C}_{2}\right)$. IR $(\mathrm{KBr}) \mathrm{cm}^{-1} 3452(\mathrm{NH}, \mathrm{st}), 1724(\mathrm{C}=\mathrm{O} s t) . \mathrm{MS}(70 \mathrm{eV}) \mathrm{m} / \mathrm{z}$ (\%) 329 (M+100), 300 (11), 272 (19), 246 (10). HR-MS calc. for $\mathrm{C}_{20} \mathrm{H}_{15} \mathrm{~N}_{3} \mathrm{O}_{2} 329.1164$, found 329.1165. Anal. Calcd for $\mathrm{C}_{20} \mathrm{H}_{15} \mathrm{~N}_{3} \mathrm{O}_{2}$ : C, 72.94; H, 4.59; N, 12.76. Found: C, 72.56; H, 4.66; N, 12.50 .

7-(4-Hydroxyphenyl)-3-methylpyrido[2,3-d]pyrimidine-2,4(1H,3H)-dione 10f. Yellow solid, recrystallized from DMF, yield 60\%, mp $>300{ }^{\circ} \mathrm{C} .{ }^{1} \mathrm{H}$ NMR (400 MHz, DMSO- $\left.d_{6}\right): \delta=3.24$ (s, 
3H, $\mathrm{NCH}_{3}$ ), 6.89 (d, 2H, $\mathrm{H}_{m}, J=8.28 \mathrm{~Hz}$ ), 7.70 (d, 1H, $\mathrm{H}_{6}, J=8.28 \mathrm{~Hz}$ ), 8.02 (d, 2H, $\mathrm{H}_{o}, J=$ $8.78 \mathrm{~Hz}), 8.24$ (d, $\left.1 \mathrm{H}, \mathrm{H}_{5}, J=8.28 \mathrm{~Hz}\right), 11.84(\mathrm{~s}, 1 \mathrm{H}, \mathrm{NH}) .{ }^{13} \mathrm{C}$ NMR $\left(100 \mathrm{MHz}, \mathrm{DMSO}-d_{6}\right): \delta=$ $26.5\left(\mathrm{NCH}_{3}\right), 107.2\left(\mathrm{C}_{4 \mathrm{a}}\right), 116.1\left(\mathrm{C}_{o}\right), 114.7\left(\mathrm{C}_{6}\right), 129.5\left(\mathrm{C}_{m}\right), 128.3\left(\mathrm{C}_{i}\right), 137.8\left(\mathrm{C}_{5}\right), 150.2\left(\mathrm{C}_{7}\right)$, $151.2\left(\mathrm{C}_{8 \mathrm{a}}\right), 161.1\left(\mathrm{C}_{4}\right), 162.1\left(\mathrm{C}_{2}\right), 163.6\left(\mathrm{C}_{p}\right) . \mathrm{IR}(\mathrm{KBr}) \mathrm{cm}^{-1} 3362(\mathrm{OH} s t), 3257(\mathrm{NH} s t), 1720-$ 1666 (C=O st). MS (70 eV) m/z (\%) 269 (M+2), 169 (32), 141 (100), 110 (20), 84 (29), 68 (22), 43 (17). HR-MS calc. for $\mathrm{C}_{14} \mathrm{H}_{11} \mathrm{~N}_{3} \mathrm{O}_{3} 269.0728$ found 269.0730. Anal. Calcd for $\mathrm{C}_{14} \mathrm{H}_{11} \mathrm{~N}_{3} \mathrm{O}_{3}$ : C, 62.45; H, 4.12; N, 15.61. Found: C, 61.96; H, 4.26; N, 15.85.

7-(4-Chlorophenyl)-3-methylpyrido[2,3-d]pyrimidine-2,4(1H,3H)-dione 10h. Yellow solid, purified by washing with hot hexanes, yield $60 \%$, mp 313-315 ${ }^{\circ} \mathrm{C} .{ }^{1} \mathrm{H}$ NMR (400 $\mathrm{MHz}, \mathrm{CDCl}_{3}$ ): $\delta=3.22$ (s, 3H, $\mathrm{NCH}_{3}$ ), 7.56 (broad band, $2 \mathrm{H}, \mathrm{H}_{m}$ ), 7.78 (broad band, $1 \mathrm{H}, \mathrm{H}_{6}$ ), 8.11 (broad band, $2 \mathrm{H}, \mathrm{H}_{o}$ ), 8.29 (broad band, $1 \mathrm{H}, \mathrm{H}_{5}$ ), 11.95 (s, 1H, NH). ${ }^{13} \mathrm{C} \mathrm{NMR}\left(100 \mathrm{MHz} \mathrm{CDCl}_{3}\right): \delta=27.5$ $\left(\mathrm{NCH}_{3}\right), 108.7\left(\mathrm{C}_{4 \mathrm{a}}\right), 115.8\left(\mathrm{C}_{6}\right), 125.0\left(\mathrm{C}_{o}\right), 129.3\left(\mathrm{C}_{m}\right), 136.0\left(\mathrm{C}_{i}\right), 136.5\left(\mathrm{C}_{p}\right), 138.3\left(\mathrm{C}_{5}\right), 151.0$ $\left(\mathrm{C}_{8 \mathrm{a}}\right), 151.2\left(\mathrm{C}_{4}\right), 162.0\left(\mathrm{C}_{2}\right), 160.0\left(\mathrm{C}_{7}\right) . \mathrm{IR}(\mathrm{KBr}) \mathrm{cm}^{-1}$ 3230, (NH st), 1722, (C=O st). MS (70 eV) $m / z(\%)$ 287( $\left.\mathrm{M}^{+}, 100\right), 289 / 287\left(\mathrm{M}^{+2} / \mathrm{M}^{+}, 33 / 100\right), 258 / 256(20 / 6), 230(30), 203 / 201$ (25/6), 140 (43). HR-MS calc. for $\mathrm{C}_{14} \mathrm{H}_{10} \mathrm{ClN}_{3} \mathrm{O}_{2} 287.0462$ found 287.0459. Anal. Calcd for $\mathrm{C}_{14} \mathrm{H}_{10} \mathrm{ClN}_{3} \mathrm{O}_{2}$ : C, 58.45; H, 3.50; N, 14.61. Found: C, 58.86; H, 3.46; N, 14.85 .

7-(4-Bromophenyl)-3-methylpyrido[2,3-d]pyrimidine-2,4(1H,3H)-dione 10i. Pink solid, recrystallized from ethanol, yield 50\%, mp 310-312 ${ }^{\circ} \mathrm{C}$ dec. ${ }^{1} \mathrm{H}$ NMR $\left(400 \mathrm{MHz}, \mathrm{CDCl}_{3}\right): \delta=$ 3.47 (s, 3H, $\mathrm{NCH}_{3}$ ), $7.59-7.64$ (m, 3H, $\mathrm{H}_{m}, \mathrm{H}_{6}$ ), 7.92 (d, 2H, $\left.\mathrm{H}_{o}, J=7.53 \mathrm{~Hz}\right), 8.12$ (s, 1H, NH), 8.45 (d, $\left.1 \mathrm{H}, \mathrm{H}_{5}, J=8.03 \mathrm{~Hz}\right) .{ }^{13} \mathrm{C}$ NMR (100 MHz, $\left.\mathrm{CDCl}_{3}\right): \delta=27.5\left(\mathrm{OCH}_{3}\right), 99.7\left(\mathrm{C}_{4 \mathrm{a}}\right), 115.7$ $\left(\mathrm{C}_{6}\right), 128.7\left(\mathrm{C}_{m}\right), 131.9\left(\mathrm{C}_{o}\right), 138.3\left(\mathrm{C}_{5}\right)$. IR $(\mathrm{KBr}) \mathrm{cm}^{-1} 3193(\mathrm{NH} s t), 1733(\mathrm{C}=\mathrm{O} s t)$. MS (70 eV) $m / z$ (\%) 332/330 (M $\left.\mathrm{M}^{+2} / \mathrm{M}^{+}, 97 / 100\right), 303 / 301(15 / 15), 275 / 273(25 / 23), 248 / 246(20 / 22), 140$ (72). HR-MS calc. for $\mathrm{C}_{14} \mathrm{H}_{10} \mathrm{BrN}_{3} \mathrm{O}_{2} 330.9956$ found 330.9970. Anal. Calcd for $\mathrm{C}_{14} \mathrm{H}_{10} \mathrm{BrN}_{3} \mathrm{O}_{2}$ : C, 50.62; H, 3.03; N, 12.65. Found: C, 50.22; H, 3.28; N, 12.74.

7-(4-Fluorophenyl)-3-methylpyrido[2,3-d]pyrimidine-2,4(1H,3H)-dione 10j. Yellow solid, yield 60\%, mp 295-297 ${ }^{\circ} \mathrm{C}$. ${ }^{1} \mathrm{H}$ NMR (400 MHz, $\left.\mathrm{CDCl}_{3}\right): \delta=3.21$ (s, 3H, $\mathrm{NCH}_{3}$ ), $7.32(\mathrm{t}, 2 \mathrm{H}$, $\mathrm{H}_{m}$ ), 7.75 (d, $1 \mathrm{H}, \mathrm{H}_{6}, J=8.28 \mathrm{~Hz}$ ), $8.13-8.17$ (m, 2H, $\mathrm{H}_{o}$ ), 8.27 (d, $\left.1 \mathrm{H}, \mathrm{H}_{5}, J=8.28 \mathrm{~Hz}\right), 11.95$ (s, 1H, NH). ${ }^{13} \mathrm{C}$ NMR (100 MHz, $\left.\mathrm{CDCl}_{3}\right): \delta=27.6\left(\mathrm{NCH}_{3}\right), 108.3\left(\mathrm{C}_{4 \mathrm{a}}\right), 115.6\left(\mathrm{C}_{6}\right), 116.4\left(\mathrm{C}_{m}\right)$, $130.1\left(\mathrm{C}_{o}\right), 133.9\left(\mathrm{C}_{i}\right), 138.3\left(\mathrm{C}_{5}\right), 151.2\left(\mathrm{C}_{4}\right), 162.1\left(\mathrm{C}_{2}\right), 162.7\left(\mathrm{C}_{8 \mathrm{a}}\right), 165.8\left(\mathrm{C}_{p}\right) . \mathrm{IR}(\mathrm{KBr}) \mathrm{cm}^{-1}$ 3222 (NH, st), 1717 (C=O st). MS (70 eV) m/z (\%) $271\left(\mathrm{M}^{+}, 100\right), 242$ (19), 214 (22), 187 (19), 158 (18), 69 (27). HR-MS calc. for $\mathrm{C}_{14} \mathrm{H}_{10} \mathrm{FN}_{3} \mathrm{O}_{2}$ 271.0757, found 271.0752. Anal. Calcd for $\mathrm{C}_{14} \mathrm{H}_{10} \mathrm{FN}_{3} \mathrm{O}_{2}$ : C, 61.99; H, 3.72; N, 15.49. Found: C, 61.22; H, 3.46; N, 15.50.

\section{Acknowledgements}

Authors wish to thank COLCIENCIAS, Universidad del Valle, Universidad de Jaén (project reference UJA_07_16_33), the Spanish “Consejería de Innovación, Ciencia y Empresa, Junta de Andalucía” the "Servicios Técnicos de Investigación de la Universidad de Jaén” and Ministerio de Ciencia e Innovación (project reference SAF2008-04685-C02-02) for financial support. 


\section{References}

1. (a) Tu, S.; Li, C.; Shi, F.; Zhou, D.; Shao, Q.; Cao, L.; Jiang, B. Synthesis 2008, 3, 369. (b) Bagley, M. C.; Hughes, D. D.; Lloyd R and Powers V. E. C. Tetrahedron Lett. 2001, 42, 6585. (c) Ren, Q.-Y.; Wang, T.; Liu, J.-C.; He, H.-W. Chinese Journal of Organic Chemistry 2005, 25, 1530.

2. Devi, I.; Kumar B. S. D.; Bhuyan, P. J. Tetrahedron Lett. 2003, 44, 8307.

3. Devi, I.; Borah, H. N.; Bhuyan, P. J. Tetrahedron Lett. 2004, 45, 2405.

4. (a) Kanth, S. R.; Reddy; G. V.; Kishore, K. H.; Rao, P. S.; Narsaiah, B.; Murthy, U. S. N. Eur. J. Med. Chem. 2006, 41, 1011. (b) Bulicz, J; Bertarelli, D. C. G.; Baumert, D.; Fülle, F.; Müller, C. E.; Heber, D. Bioorg. Med. Chem. 2006, 14, 2837. (c) Tu, S.; Zhang, J.; Zhu, X.; Xu, J.; Zhang, Y.; Wang, O; Jia, R.; Jiang, B.; Zhang, J. Bioorg. Med. Chem. Lett., 2006, 16, 3578.

5. (a) El-Gazzar, A. B. A.; Hafez, H. A.; Yakout, E. M. A. J. Chin. Chem. Soc., 2007, 54, 1303.

(b) Chebanov, V. A.; Saraev, V. E.; Gura, E. A.; Desenko, S. M.; Musatov, V. I. Collect. Czech. Chem. Commun. 2005, 70, 350. (c) Ojea, V.; Muinelo, L.; Quintela, J. M. Tetrahedron 1998, 54, 927. (d) Katritzky, A. R.; Rees, C.W., Scriven, E .F. V., Eds. Comprehensive Heterocyclic Chemistry-II, Elsevier: Oxford, 1996; Vol. 7, pp 591. (e) Abdel-Hady, S. A. L.; Badawy, M. A.; Kasry, A. M.; Mosselhi, M. A. N. J. Heterocyclic Chem., 1987, 24, 1587. (f) Troschütz, R.; Roth, H. J. Arch. Pharm. 1978, 311, 406.

6. (a) Hassan, N. A.; Hegab, M. I.; Hashem, A.I.; Abdel-Motti, F. M.; Hebah, S. H. A.; AbdelMegeid, F. M. E. J. Heterocyclic Chem. 2007, 44, 775. (b) Kidwai, M.; Singhal, K. Synth. Commun. 2006, 36, 1887. (c) Mont, N.; Teixidó, J.; Borrell, J. I.; Kappe, C. O. Tetrahedron Lett. 2003, 44, 5385.

7. (a) Gustin, D.; Divirgilio, E.; Guram, A.; Faul, M. M. Synthesis 2007, 23, 3678. (b) Chan, J.; Faul, M. Tetrahedron Lett. 2006, 47, 3361.

8. Bae, J. W.; Lee, S. H.; Cho, Y. J.; Jung, Y. J; Hwang, H. J.; Yoon, C. M. Tetrahedron Lett. 2000, 41, 5899.

9. Tu, S; Cao, L.; Zhang, Y.; Shao, Q.; Zhou, D.; Li, C. Ultrason. Sonochem. 2008, 15, 217.

10. (a) Quiroga, J.; Insuasty, H.; Insuasty, B.; Abonía, R.; Cobo, J.; Sánchez, A.; Nogueras, M. Tetrahedron 2002, 58, 4873. (b) Quiroga, J.; Rengifo, A.; Insuasty, B.; Abonía, R.; Sánchez, A.; Nogueras, M. Tetrahedron Lett. 2002, 43, 9061. (c) Quiroga, J.; Insuasty, H.; Insuasty, B.; Abonía, R.; Ortíz, A.; Sánchez, A.; Nogueras, M. J. Heterocyclic Chem. 2001, 38, 339. (d) Quiroga, J.; Insuasty, B.; Abonía, R.; Hernández, P.; Nogueras, M.; Sánchez, A. Heterocyclic Commun. 2000, 6, 345. (e) Quiroga, J.; Alvarado, M.; Insuasty, B.; Nogueras, M.; Sánchez, A.; López, M. D. J. Heterocyclic Chem. 1999, 36, 113. (f) Cobo, J.; Sánchez, A.; Nogueras, M. Trends Heterocyclic Chem. 1999, 6, 97. (g) Quiroga, J.; Hormaza, A.; Insuasty, B.; Ortíz, A. J.; Sánchez, A.; Nogueras, M. J. Heterocyclic Chem. 1998, 35, 231. (h) Quiroga, J.; Hormaza, A.; Insuasty, B.; Nogueras, M.; Sánchez, A.; Hanold, N.; Meier, H. J. Heterocyclic Chem. 1997, 34, 521; (i) Quiroga, J.; García, J.; Insuasty, B.; Mendoza, N. L.; 
Pungo, M.; Meier, H. An. Quím. 1994, 90, 300. (j) Quiroga, J.; Insuasty, B.; Sánchez, A.; Nogueras, M.; Meier, H. J. Heterocyclic Chem. 1992, 29, 1045.

11. Quiroga, J.; Cisneros, C.; Insuasty, B.; Abonía, R.; Cruz, S.; Nogueras, M.; De la Torre, J. M.; Sortino, M.; Zacchino, S. J. Heterocyclic Chem. 2006, 43, 299. (b) Quiroga, J.; Cisneros, C.; Insuasty, B.; Abonía, R.; Nogueras, M.; Sortino, M.; Zacchino, S. J. Heterocyclic Chem. 2006, 43, 463.

12. (a) Yang, D.; Jiang, K.; Li, J.; Xu, F. Tetrahedron 2007, 63, 7654. (b) Zong, R.;Wang, D.; Hammitt, R.; Thummel, R. P. J. Org. Chem. 2006, 71, 167. (c) Dormer, P. G.; Eng, K. K.; Farr, R. N.; Humphrey, G.; Mcwilliams, J. C.; Reider, P. J.; Sager, J. W.; Volante, R. P. J. Org. Chem. 2003, 68, 467. (d) Marco-Contelles, J.; Pérez-Mayoral, E.; Samadi, A.; Carreiras, M. D.; Soriano, E. Chem. Rev. 2009, 109, 2652.

13. Sivaprasad, A.; Sandhu, J. S.; Baruah, J. N. Indian J. Chem. 1985, 24B, 305.

14. Pfleiderer, W.; Strauss, G. Liebigs Annalen 1957, 612, 173.

15. (a) Delia, T. J.; Otteman, R. Heterocycles 1983, 20(9), 1805. (b) Negrillo, J.; Nogueras, M.; Sánchez, A.; Melgarejo, M. Chem. Pharm. Bull. 1988, 36, 386. (c) Erkin, A. V.; Krutikov, V. I. Russ. J. Gen. Chem. 2004, 74, 146.

16. Sivaprasad, A.; Sandhu, J. S.; Baruah, J. N. Indian J. Chem. 1985, 24B, 305.

17. (a) Cherdantseva, N. M.; Nesterov, V. M.; Safonova, T. S. Khim. Geterotsikl. Soedin. 1983, 6, 834. (b) de la Torre, J. M.; Trilleras, J.; Cobo, J.; Glidewell, C. Acta Crystallogr. Sect C 2009, C65, 281.

18. Nerender, T.; Reddy, K. P. Tetrahedron Lett. 2007, 48, 3177.

19. (a) Quiroga, J.; Viveros, G.; Insuasty, B.; Nogueras, M.; Sánchez, A.; Cobo, J. J. Heterocyclic Chem. 1999, 36, 501. (b) Salas, P. J. M.; Moreno, C. M.; Nogueras, M. M.; Sánchez, R. A. An. Quím. B, 1983, 79, 547.

20. Trilleras, J.; Quiroga, J.; Cobo, J.; Glidewell, C. Acta Crystallogr. Sect C. 2009, C65, 134. 